\title{
Immiscible Thermo-Viscous Fingering in Hele-Shaw cells
}

\author{
S.J. Jackson, H. Power*, D. Giddings \\ Fluids and Thermal Engineering Research Group, University of Nottingham, UK
}

\begin{abstract}
We investigate immiscible radial displacement in a Hele-Shaw cell with a temperature dependent viscosity using two coupled high resolution numerical methods. Thermal gradients created in the domain through the injection of a low viscosity fluid at a different temperature to the resident high viscosity fluid can lead to the formation of unstable thermo-viscous fingers, which we explore in the context of immiscible flows. The transient, multi-zone heat transfer is evaluated using a newly developed auxiliary radial basis function-finite collocation (RBF-FC) method, which locally captures variation in flux and field variable over the moving interface, without the need for ghost node extrapolation. The viscosity couples the transient heat transfer to the Darcy pressure/velocity field, which is solved using a boundary element - RBF-FC method, providing an accurate and robust interface tracking scheme for the full thermo-viscous problem.

We explore the thermo-viscous problem space using systematic numerical experiments, revealing that the early stage finger growth is controlled by the pressure gradient induced by the varying temperature and mobility field. In hot injection regimes, negative temperature gradients normal to the interface act to accelerate the interface, promoting finger bifurcation and enhancing the viscous fingering instability. Correspondingly, cold injection regimes stabilise the flow compared to isothermal cases, hindering finger formation. The interfacial mobility distribution controls the late stage bifurcation mode, with non-uniformities induced by the thermal diffusivity creating alternate bifurcation modes. Further numerical experiments reveal the neutral stability of the thermal effects on the fingering evolution, with classical viscous fingering dynamics eventually dominating the evolution. We conclude the paper with a mechanistic summary of the immiscible thermo-viscous fingering regime, providing the first detailed analysis of the thermal problem in immiscible flows.
\end{abstract}

Keywords: , Thermo-Viscous Fingering, Immiscible, RBF, BEM

\section{Introduction}

During the displacement of a high viscosity fluid by the injection of a low viscosity fluid, interfacial instabilities can evolve forming complex viscous fingering patterns. The viscous fingering mechanism is an important feature in many practical applications, particular those involving the flow through porous media, such as enhanced oil recovery [1], geothermal heat extraction [2] and carbon sequestration [3] (for a detailed review of viscous fingering in porous media see [4]).

One such porous media flow, and the primary motivation behind the current work is the injection and storage of supercritical $\mathrm{CO}_{2}$ in deep subsurface aquifers (carbon sequestration). When $\mathrm{CO}_{2}$ is injected into a deep saline geological formation suitable for storage, it will displace the resident brine, creating an immiscible two-phase flow regime. The injected $\mathrm{CO}_{2}$ will rise upwards due to buoyancy forces, eventually hitting a low permeability cap rock where a permeability and capillary barrier stops the $\mathrm{CO}_{2}$ from rising out of the aquifer. After this stage, the $\mathrm{CO}_{2}$ will spread laterally, further displacing brine.

The injection of supercritical $\mathrm{CO}_{2}$ will typically occur at a temperature different to that of the resident brine, creating temperature gradients in the domain that will alter mechanical fluid

\footnotetext{
* Corresponding author

Email address: henry.power@nottingham.ac.uk (H. Power)
} 
quantities such as diffusivity, viscosity, density and surface tension. The $\mathrm{CO}_{2}$ can be injected at a colder temperature than the resident brine, due to heat loss en-route to the downhole injection site [5], or through very deep subsurface injection where the resident brine temperature is very high (typically at depths $>3 \mathrm{~km}$ ), e.g. the In Salah formation in Algeria [6]. $\mathrm{CO}_{2}$ can also be used for combined storage and extraction in geothermal reservoirs, in which very high temperature brine $\left(\mathrm{T}>150^{\circ} \mathrm{C}\right)$ can be extracted for energy use [7]. The $\mathrm{CO}_{2}$ may also be injected at a higher temperature than the resident brine, for pre-conditioning purposes [8] or for plume evolution monitoring [9]. Another immiscible displacement regime where thermal effects are prominent is that of thermal enhanced oil recovery, in which high temperature water (or steam) is used to mobilise trapped oil and increase well production [10].

Understanding how temperature gradients affect the fluid properties and resulting interfacial displacement is key to understanding the key physical mechanisms in the immiscible displacement regimes above and in the fundamental study of moving multi-zone problems in classical fluid dynamics. In this work, we analyse the changes in fluid viscosity due to thermal gradients in the domain and the effect this has on the immiscible interfacial displacement and viscous fingering process, often termed thermo-viscous fingering.

In order to examine the effects coming purely from the variable viscosity, we maintain constant values of surface tension and diffusivity for each fluid, with density driven buoyancy effects negligible in the lateral fluid flow. This simplification allows the thermo-viscous fingering process to be quantified for immiscible regimes without the complication of additional Marangoni and variable diffusivity effects. To analyse the fully coupled thermal problem involves the solution of a different set of non-linear equations requiring inclusion of viscous shear stresses around the interface and a non-linear diffusion term in the heat transfer. The solution of these coupled equations would require more complex numerical techniques that are beyond the scope of this work. We direct the interested reader towards the following works that focus on the other thermal processes mentioned above: [11, 12] for Marangoni type effects and [13] for the solution of non-linear diffusivity problems.

In order to model the immiscible displacement regimes we use the analogy between Hele-Shaw and porous media flows. By considering that the flow between the plates in the Hele-Shaw cell follows a Poiseuille profile, Stokes flow can be reduced to Darcy flow by depth-averaging across the gap. The immiscible displacement of the fluids is then described by 2D potential flow in the plane of the Hele-Shaw cell. As the thermal diffusivities of the two fluids can be very low, the thermal Peclet number describing the ratio of convective to diffusive heat transfer can be large $(>1000)$. We therefore have to solve the full convection-diffusion heat equation to track the heat transfer in the domain.

During thermo-viscous fingering, generally, two fronts are established in the domain: 'solutal' and thermal. The solutal front is defined here as the point at which the fluid composition transitions from the injected fluid to the resident fluid. In immiscible displacement this is a sharp front controlled by capillary forces, where the mechanical fluid properties exhibit discontinues profiles [14]. The thermal front in an immiscible regime is also 'sharp' if the fluids have different diffusivities, creating a defined multi-zone domain.

The solutal and thermal fronts do not necessarily propagate with the same velocity due to a difference in solutal and thermal diffusivities and the fact that heat may diffuse away from the fluid into surrounding solid media. In the case of a thermally insulated Hele-Shaw cell, there is no thermal lag, as the cell remains an open channel with an effective porosity of one. The solutal and thermal fronts advance with the same velocity, creating a single or iso-advective regime, that is governed purely by the fluid velocity [15]. Also, due to the immiscibility of the fluids, we only consider thermal diffusivity (i.e there is no mass diffusivity), meaning double diffusive effects apparent in miscible flows are not encountered here [16].

There exists a very limited number of studies on immiscible thermo-viscous fingering, with most previous research focusing on miscible cases. One notable work in the immiscible regime by Sheorey and Muralidhar finds that non-isothermal, high temperature injection of water into resident oil promotes the formation of viscous fingers and increases the sweep efficiency of the water [17]. The fluid front can temporarily displace beyond the thermal front into a region of low temperature (and high viscosity), where the front velocity drops. The sides of the front can then bypass this region, forming fingers around the sides of the domain. The finger evolution here 
is a result of the interplay between the thermal and solutal fronts, which despite the exclusion of a thermal lag parameter, have different diffusive regimes and hence affect the advancing front on different time scales. It is also worth noting, that due to the constitutive relationship for the capillary pressure based on the water saturation alone (without temperature variation), and the Darcy flow approximation [17], thermocapillary and Marangoni effects are not included in their model, as is the case in the present work.

The interplay between solutal and thermal fronts has been explored in much more detail in the context of misicible flows. In the analysis of radial double-diffusive fronts, Pritchard finds that even for strongly stabilising temperature fields (i.e. they form a decreasing viscosity profile across the interface), a destabilising viscosity profile can be maintained over the front due to a strongly advective solute regime [14], unless the thermal lag is near unity and the thermal viscosity contrast is many times higher than the viscosity contrast across the solutal front. Mishra et al. [16] and Pritchard [15] show that the aggregate viscosity profile is not sufficient to predict the overall stability at late stages, as diffusive effects become more prominent, and early stage stabilisation of the flow may diminish. It is not enough to state that if one of the scalar components promotes fingering, then fingering will eventually occur in the late stage regime [15].

Numerical studies of various miscible displacements regimes agree qualitatively with the stability analyses above $[2,18,19,20,21]$. In general, at the late stages of interfacial growth, they find that at sufficiently low values of thermal lag, or if the thermal regime is highly diffusive, the evolution depends entirely on the solutal viscosity ratio. The (de)stabilising effects of the thermal front are reduced with an increase in thermal diffusivity or thermal lag [20, 18].

Experimentally, thermo-viscous fingering is typically hard to quantify under laboratory conditions. Nagatsu et al. [22] and Holloway and de Bruyn [23] track the injection of hot glycerine (glycerine-water solutions in [22]) into colder more viscous glycerine in a Hele-Shaw cell. Nagatsu et al. [22] find that in a small range of mobility ratios, around $\beta=M_{1} / M_{2}=10$, injection at a higher temperature than the resident fluid promotes the onset of fingering, with bifurcation occurring at a smaller interface radius. The results have good agreement with Holloway and de Bruyn [23]. However, more specific thermal effects are hard to gauge due to the insulation of the Hele-Shaw cell and resulting heat loss through the glass plates [22].

In this current work, we analyse the immiscible thermo-viscous fingering process in a Hele-Shaw cell due to the radial injection of a low viscosity fluid with injection temperature different to that of the high viscosity resident fluid. We use a Hele-Shaw model with efficient interface tracking methods to study the effects of thermo-viscous fingering in an immiscible regime, allowing the basic mechanisms of the thermo-viscous process to be examined in detail. Using this simplified model, we hope to shed light on the more complex problem occurring in porous media. To the authors knowledge, this work represents the first comprehensive numerical study of immiscible thermo-viscous fingering in a Hele-Shaw cell, and hopes to shed light on the effects of multi-zone heat transfer on the purely advective immiscible fluid flow regime.

Using a recently developed boundary element (BE) - radial basis function (RBF) method, we track the fluids' pressure, velocity and temperature through the solution of Darcy flow and the multi-zone convection-diffusion of heat through the domain. This method allows the late stage exploration of the effects of a transient temperature field on the immiscible fluid front, and the resulting displacement regime. The paper is organised as follows; firstly, we present the mathematical model followed by a summary of the numerical methods. After this we present results of immiscible thermo-viscous fingering under different capillary number, temperature and diffusivity regimes, concluding with a discussion and summary of the results.

\section{Mathematical formulation}

We consider a radial Hele-Shaw cell, in which high viscosity fluid is displaced by the radial injection of a low viscosity fluid. The low viscosity invading fluid (such as $\mathrm{CO}_{2}$ ) with temperature $T_{0}$ occupies the inner region $\Omega_{1}$, whilst a high viscosity fluid (such as brine) with temperature $T_{\infty}$ occupies the external region $\Omega_{2}$, shown in Figure 1. The interface separating the internal and external region has an initial perturbation given by $r=r_{0}+\epsilon_{0} r_{0} \cos (8 \theta)$, where $r, r_{0}, \epsilon_{0}$ and $\theta$ are the interface radius, the unperturbed interface radius, the perturbation amplitude and 


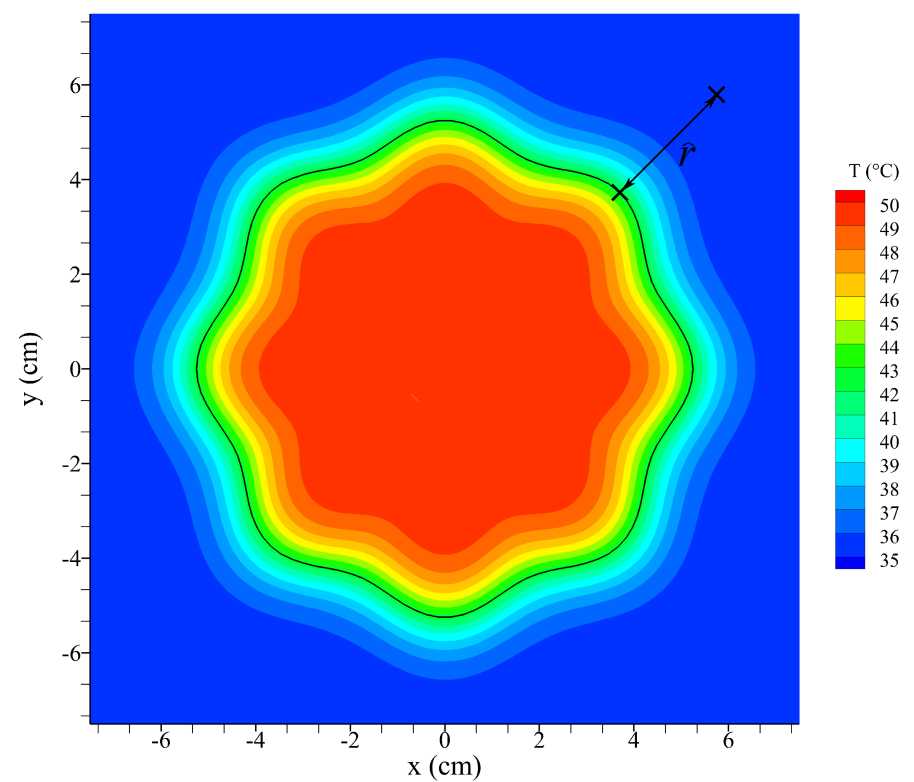

Figure 1: Initial setup of the immiscible thermo-viscous fingering problem in a Hele-Shaw cell. The black fluid-fluid interface overlays a contour plot of the initial temperature field. The radial distance used in the smoothed step function $\hat{r}$ is highlighted.

the azimuthal angle around the interface respectively. The initial temperature $T(\boldsymbol{x})$ at a point $\boldsymbol{x}=\left(x_{1}, x_{2}\right)$ is given by a smoothed step function, i.e.

$$
T(\boldsymbol{x})=T_{\infty}+0.5\left(T_{0}-T_{\infty}\right)\left(1+\tanh \left(\frac{\hat{r}}{\delta}\right)\right)
$$

where, $\hat{r}$ is the radial distance from a point $\boldsymbol{x}$ to the corresponding interface point. $\delta$ is the sharpness of the smoothed step function. We use a smoothed step function to aid numerical stability at the start of simulations when the velocity is very high. An initially discontinuous temperature field can be used, but the late stage finger growth (and temperature field) only shows very minor differences with that computed from an initially smooth temperature field.

For the flow in a Hele-Shaw cell, the depth averaged pressure $P_{l}$ and 2D Darcy velocity $u_{i}^{l}$ in each fluid region $l$ can be expressed through Darcy's law: $u_{i}^{l}(\boldsymbol{x})=-M_{l}(\boldsymbol{x}) \frac{\partial P_{l}}{\partial x_{i}}$. The mobility $M_{l}$ is related to the fluid viscosity $\mu_{l}(\boldsymbol{x})$ and plate spacing $b$ by: $M_{l}(\boldsymbol{x})=b^{2} / 12 \mu_{l}(\boldsymbol{x})$. Substituting the Darcy flow expression into the conversation of mass, we find:

$$
\frac{\partial}{\partial x_{i}}\left(M_{l}(\boldsymbol{x}) \frac{\partial P_{l}(\boldsymbol{x})}{\partial x_{i}}\right)=0 \quad \boldsymbol{x} \in \Omega_{l}, \quad l=1,2
$$

where $M_{l}$ is a function of space and time since $\mu_{l}$ is a function of the transient temperature field. The plate spacing $b$ has been dropped from the equation since it is constant in this formulation. At a boundary point $\boldsymbol{\xi}$ on the fluid interface $S$ between $\Omega_{1}$ and $\Omega_{2}$, there are two matching conditions (kinematic and dynamic) that must be met by the advancing interface. Firstly, the kinematic condition requires the continuity of normal velocity $u_{i}(\boldsymbol{\xi}) n_{i}(\boldsymbol{\xi})$, i.e.

$$
-M_{1}(\boldsymbol{\xi}) \frac{\partial P_{1}(\boldsymbol{\xi})}{\partial n}=-M_{2}(\boldsymbol{\xi}) \frac{\partial P_{2}(\boldsymbol{\xi})}{\partial n}
$$

Secondly, the dynamic condition describes the pressure jump across the interface:

$$
P_{1}(\boldsymbol{\xi})-P_{2}(\boldsymbol{\xi})=\gamma\left(\frac{2}{b}+\frac{\pi}{4} k(\boldsymbol{\xi})\right)
$$


where $\gamma$ is the surface tension and $k(\boldsymbol{\xi})$ is the curvature. $k(\boldsymbol{\xi})$ is considered a continuous function on the interface surface with a $\pi / 4$ scaling term included for consistency with the asymptotic analysis of [24]. The contact angle of the meniscus has been assumed to be zero and we neglect dynamic wetting effects. Dynamic wetting has been shown by various authors to have a considerable effect on the interfacial displacement in a Hele-Shaw cell at high capillary numbers and requires study in its own right, putting it beyond the scope of the current work $([25,26,27])$. The displacement of the outer fluid is initiated by the injection of the inner fluid with a point source of strength $Q$ at the origin.

In order to characterise effects coming from the non-isothermal regime, we decompose the variables that relate to the viscosity into homogeneous and perturbed components. Firstly, the viscosity of each fluid $l$ can be represented through an exponential dependence on the temperature[14][28].

$$
\mu_{l}(\boldsymbol{x})=\overline{\mu_{l}} \exp \left[\mp \alpha_{l}\left(1 \pm \frac{T(\boldsymbol{x})-T_{\infty}}{\left|T_{0}-T_{\infty}\right|}\right)\right] \quad \boldsymbol{x} \in \Omega_{l}, \quad l=1,2
$$

Here, the homogeneous viscosity $\overline{\mu_{l}}$ is that of fluid $l$ at the injection temperature $T_{0} . T_{\infty}$ is the resident fluid temperature, $\alpha_{l}$ is the rate of change of viscosity for fluid $l$ with temperature. The minus/plus sign in $\mp \alpha_{l}$ is used for when the injecting fluid is hotter $(+)$ or colder $(-)$ than the resident fluid. This gives the mobility of the fluid in zone $l$ as:

$$
\begin{array}{r}
M_{l}(\boldsymbol{x})=\frac{b^{2}}{12 \mu_{l}(\boldsymbol{x})}=\bar{M}_{l}+\tilde{M}_{l}(\boldsymbol{x}) \\
\bar{M}_{l}=\frac{b^{2}}{12 \overline{\mu_{l}}}, \quad \tilde{M}_{l}(\boldsymbol{x})=\frac{b^{2}}{12 \mu_{l}(\boldsymbol{x})}-\bar{M}_{l}
\end{array}
$$

We now introduce non-dimensional variables utilising the characteristic length, time, velocity, pressure, temperature and mobility of the problem, given by:

$$
\begin{array}{r}
(\boldsymbol{x}, r, b)=r_{0}\left(\boldsymbol{x}^{\prime}, r^{\prime}, b^{\prime}\right), \quad t=\frac{r_{0}^{2}}{Q} t^{\prime}, \\
M_{l}=\bar{M}_{2} M_{l}^{\prime}, \quad u_{l}=\frac{Q}{r_{0}} u_{l}^{\prime}, \quad P_{l}=\frac{Q}{\bar{M}_{2}} P_{l}^{\prime}, \\
T_{l}=\left|T_{0}-T_{\infty}\right| T_{l}^{\prime}+T_{\infty}, \quad D_{l}=Q D_{l}^{\prime} \quad l=1,2
\end{array}
$$

In equalities (8) - (10), apostrophes identify dimensionless variables with $t$ as time and $\bar{M}_{2}$ as the homogeneous mobility of the displaced fluid (corresponding to the homogeneous viscosity $\overline{\mu_{2}}$ ). $D_{l}$ is the thermal diffusivity of fluid $l$.

From this point onwards, the apostrophe of all non-dimensional variables will be dropped, and all variables will be assumed to be in their non-dimensional form unless otherwise stated. We start by representing the pressure as a sum of homogeneous and perturbed components:

$$
P_{l}(\boldsymbol{x})=\bar{p}_{l}(\boldsymbol{x})+\tilde{p}_{l}(\boldsymbol{x})
$$

Using the above form of the pressure, equation (2) can be expanded, noting that $\partial \bar{M}_{l} / \partial x_{i}=0$, to obtain the following equation:

$$
M_{l}(\boldsymbol{x}) \frac{\partial^{2} \bar{p}_{l}}{\partial x_{i} \partial x_{i}}+M_{l}(\boldsymbol{x}) \frac{\partial^{2} \tilde{p}_{l}(\boldsymbol{x})}{\partial x_{i} \partial x_{i}}+\frac{\partial \tilde{M}_{l}(\boldsymbol{x})}{\partial x_{i}} \frac{\partial \tilde{p}_{l}(\boldsymbol{x})}{\partial x_{i}}=-\frac{\partial \tilde{M}_{l}(\boldsymbol{x})}{\partial x_{i}} \frac{\partial \bar{p}_{l}(\boldsymbol{x})}{\partial x_{i}}
$$

where the homogeneous pressure is constrained to satisfy Laplace's equation, in order to characterise the case of isothermal displacement, i.e.:

$$
\frac{\partial^{2} \bar{p}_{l}(\boldsymbol{x})}{\partial x_{i} \partial x_{i}}=0
$$


The homogeneous pressure is then subject to the following matching and asymptotic conditions:

$$
\begin{aligned}
\bar{p}_{1}(\boldsymbol{\xi})-\bar{p}_{2}(\boldsymbol{\xi}) & =\frac{1}{C a_{g}}\left(\frac{2}{b}+\frac{\pi}{4} k(\boldsymbol{\xi})\right) \quad \text { for } \quad \boldsymbol{\xi} \in S \\
\bar{M}_{1}(\boldsymbol{\xi}) \frac{\partial \bar{p}_{1}(\boldsymbol{\xi})}{\partial n} & =\bar{M}_{2}(\boldsymbol{\xi}) \frac{\partial \bar{p}_{2}(\boldsymbol{\xi})}{\partial n} \quad \text { for } \quad \boldsymbol{\xi} \in S \\
\bar{p}_{2}(\boldsymbol{x}) & \rightarrow-\frac{1}{2 \pi} \ln (r)
\end{aligned}
$$

The global capillary number $C a_{g}$ in (14) describes the ratio of viscous driving forces to surface tension forces in terms of the characteristic mobility $\bar{M}_{2}$ and plate separation $b$ at the origin; $C a_{g}=r_{0} Q / \gamma \bar{M}_{2}$. The perturbed pressure equation (12) is then subject to the following matching conditions at the interface, $S$, and asymptotic conditions near the origin and in the far field:

$$
\begin{aligned}
\tilde{p}_{1}(\boldsymbol{\xi})-\tilde{p}_{2}(\boldsymbol{\xi}) & =0 \quad \text { for } \quad \boldsymbol{\xi} \in S \\
M_{1}(\boldsymbol{\xi}) \frac{\partial \tilde{p}_{1}(\boldsymbol{\xi})}{\partial n} & =M_{2}(\boldsymbol{\xi}) \frac{\partial \tilde{p}_{2}(\boldsymbol{\xi})}{\partial n}+\left(\beta \tilde{M}_{2}(\boldsymbol{\xi})-\tilde{M}_{1}(\boldsymbol{\xi})\right) \frac{\partial \bar{p}_{1}(\boldsymbol{\xi})}{\partial n} \quad \text { for } \quad \boldsymbol{\xi} \in S \\
\frac{\partial \tilde{p}_{1}(\boldsymbol{x})}{\partial r} & \rightarrow 0 \quad \begin{array}{l}
\tilde{p}_{2}(\boldsymbol{x}) \rightarrow 0 \\
\boldsymbol{x} \rightarrow \infty
\end{array}
\end{aligned}
$$

where $\beta$ is the mobility ratio between the fluids, $\beta=\bar{M}_{1} / \bar{M}_{2}=\bar{\mu}_{2} / \bar{\mu}_{1}$. The jump in flux in equation 18 is given by:

$$
\left(\beta \tilde{M}_{2}(\boldsymbol{\xi})-\tilde{M}_{1}(\boldsymbol{\xi})\right) \frac{\partial \bar{p}_{1}(\boldsymbol{\xi})}{\partial n}=\frac{b^{2}}{12 \bar{\mu}_{1}}\left(e^{ \pm \alpha_{2}(1 \pm T)}-e^{ \pm \alpha_{1}(1 \pm T)}\right) \frac{\partial \bar{p}_{1}(\boldsymbol{\xi})}{\partial n}
$$

Therefore, if $\alpha_{1}=\alpha_{2}$ the flux in equation (18) is continuous, however, in all cases the interfacial flux (3) is always satisfied, i.e. continuous. This can be observed by adding equations (15) and (18) and using the definitions (6) and (11) with $\beta=\bar{M}_{1} / \bar{M}_{2}$, which is valid for any value of $\alpha_{1}$ and $\alpha_{2}$. The obtained values of $\bar{p}_{l}$ and $\tilde{p}_{l}$ can be used to reconstruct the normal velocity at an interface point, $\boldsymbol{\xi}$, in order to track the fluid:

$$
U_{n}(\boldsymbol{\xi})=\bar{u}_{n}(\boldsymbol{\xi})+\tilde{u}_{n}(\boldsymbol{\xi})=-M^{1}(\boldsymbol{\xi}) \frac{\partial \bar{p}_{1}(\boldsymbol{\xi})}{\partial n}-M^{1}(\boldsymbol{\xi}) \frac{\partial \tilde{p}_{1}(\boldsymbol{\xi})}{\partial n}
$$

To evaluate the flow and pressure in the domain and calculate the interfacial velocity with equation (21) we require the temperature throughout in order to calculate the viscosity and its spatial derivatives. To track the temperature evolution through the domain, we solve the multi-zone convection diffusion heat equation:

$$
\frac{\partial T_{l}(\boldsymbol{x}, t)}{\partial t}=D_{l} \frac{\partial^{2} T_{l}(\boldsymbol{x}, t)}{\partial x_{i}^{2}}-u_{i}(\boldsymbol{x}, t) \frac{\partial T_{l}(\boldsymbol{x}, t)}{\partial x_{i}} \quad l=1,2
$$

Equation (22) is valid in each fluid region $l$, subject to the following matching, boundary and initial conditions:

$$
\begin{aligned}
T_{1}(\boldsymbol{\xi}, t) & =T_{2}(\boldsymbol{\xi}, t) \quad \text { for } \quad \boldsymbol{\xi} \in S \\
D_{1} \frac{\partial T_{1}(\boldsymbol{\xi}, t)}{\partial n} & =D_{2} \frac{\partial T_{2}(\boldsymbol{\xi}, t)}{\partial n} \quad \text { for } \quad \boldsymbol{\xi} \in S \\
T_{1}(\boldsymbol{x}, t) & \rightarrow T_{0} \quad \begin{array}{c}
T_{2}(\boldsymbol{x}, t) \rightarrow T_{\infty} \\
\boldsymbol{x} \rightarrow 0
\end{array} \\
T_{l}(\boldsymbol{x}, 0) & =f_{l}(\boldsymbol{x}) \quad \boldsymbol{x} \in \Omega_{l}, \quad l=1,2
\end{aligned}
$$

In equations (23) and (24), we enforce continuity of field variable and flux respectively, noting that the fluid velocity $u_{i}$ is continuous across the interface. The temperature follows a prescribed, smoothed step initial condition given by equation (1).

The heat transfer completes the mathematical formulation of the problem. In order to evaluate 
the pressure field in equation (12) and (13), along with the temperature field in equation (22), we require the discretisation of the domain, interface and boundaries as well as the use of suitable numerical methods, discussed in the following sections.

\section{Numerical methods}

Here, we summarise the solution procedure to evaluate the transient temperature field and quasi-static pressure in order to reconstruct the interface velocity with equation (21).

The quasi-static pressure (both homogeneous (13) and perturbed (12) components) is solved using a coupled boundary element - radial basis function - finite collocation (BE-RBF-FC) method, presented in [29]. The solution technique is the same in this paper, with changes only in the spatial form of the mobility, which is now evaluated from the transient temperature field. The BEM and RBF-FC have also been used separately in several previous works, see [27, 30] and [31, 32] respectively.

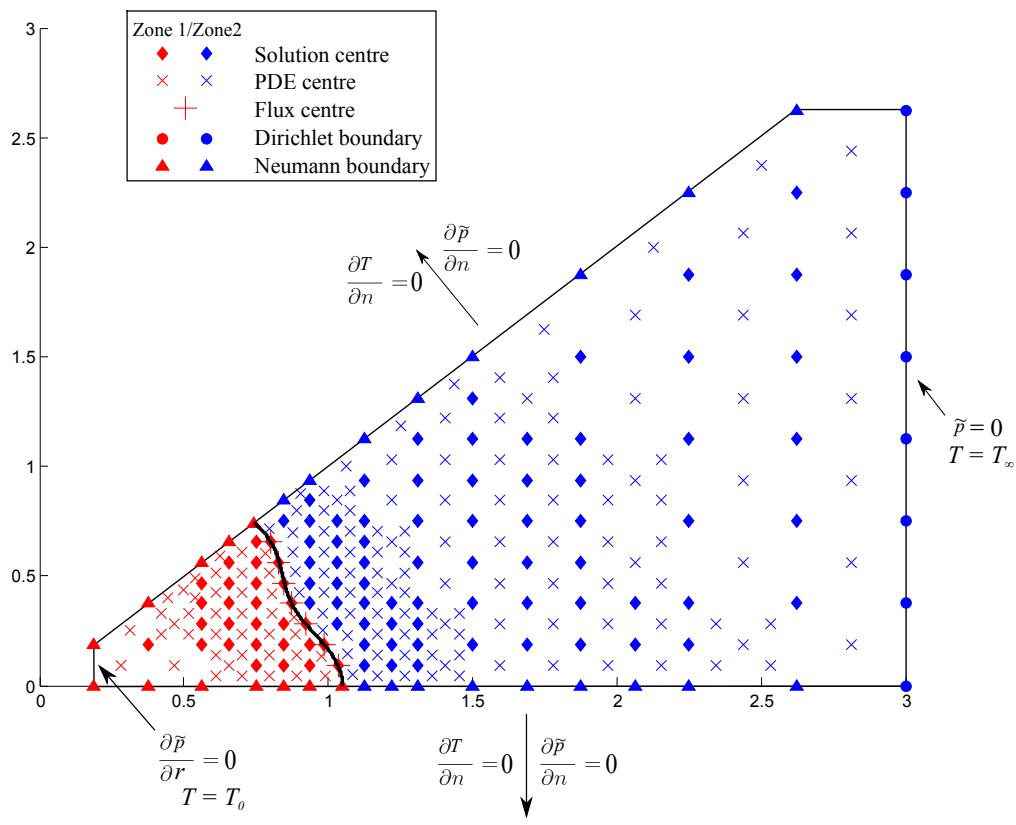

Figure 2: Thermo-viscous solution domain showing different RBF-FC operator types. The curved solid black line indicates the fluid-fluid interface.

The homogeneous pressure (13) is solved using an indirect boundary element method which explicitly tracks the interface, providing greater accuracy compared to front capturing methods, at the expense of increased meshing computational complexity. Using a convergent (Neumann) series technique, the computational cost scales quadratically with the number of boundary elements, significantly reducing running times compared with traditional $L U$ solvers of fully populated BEM matrices. As the surface interface grows, the number of boundary elements are adaptively increased to maintain a target element size.

The perturbed pressure equation (12) is solved using the embedded multi-zone RBF-FC method, originally developed in [33]. A quadtree dataset is used, to adaptively add and remove points from the domain as the interface displaces, which utilises a distance based splitting scheme. The quadtree dataset was first utilised with the RBF-FC method in [32], and later applied to multizone problems in [29]. To decrease the solution cost for this method we use an 8 finger symmetric interface throughout the numerical simulations; this enables the RBF-FC method to make use of symmetry in the domain and solve only $1 / 8^{t h}$ of the entire domain. We displace nodes to coincide directly with the interface, at which two RBF stencils can be formed to enforce the multi-zone flux and solution matching conditions.

We note that any form of symmetric could be used here to reduce computation time, but the conclusions drawn will be the same. A change in wavelength and wavenumber of initial perturbation 
will alter the magnitude and time-scale at which fingering events occur, but the mechanisms for fingering (and stability) will remain unchanged. We choose an 8 finger symmetry, since it is efficient to split a quadtree dataset into eighths, as opposed to a 6 finger symmetry which would require displacement of nodes in the quadtree dataset so that they fall onto the line of symmetry.

An example RBF-FC nodal distribution with corresponding boundary conditions is shown in Figure 2. Only $1 / 8^{\text {th }}$ of the boundary element interface is shown, although in reality the entire closed interface is solved at each time step (using symmetry to copy mobility data to parts of the interface not shown).

To solve the transient heat transfer equation (22), the RBF-FC method utilises an auxiliary multi-zone representation that enforces the interface matching conditions locally, so that ghostnode extrapolation can be avoided in the fully transient moving interface problem. We use a $2^{\text {nd }}$ order Crank-Nicholson time stepping scheme $(\theta=0.5)$ in this work. Details of the new auxiliary method, along with numerical validation are presented in the appendix.

The main difference between the auxiliary multi-zone method and the embedded method presented in previous works, is the interface representation. In the embedded method, the matching conditions appear globally, and the governing PDE is enforced locally at the interface (without local PDE points on the interface the resulting solution is highly inaccurate). For moving interface problems the embedded method therefore requires some form of extrapolation for the PDE value (from the internal zone) at the interface. However, in the auxiliary method, the matching conditions appear locally, meaning PDE points don't have to be placed on the interface for solution accuracy, and extrapolation can be alleviated.

The issue of extrapolation also occurs for internal PDE points over which the interface passes in a single time step, meaning the zone switches from 2 to 1 between time step $n$ and $n+1$. This means the value of previous time step data needs to be calculated for the point, but at time step $n$ the point was positioned in zone 2 ; no data exists at that location for zone 1 at time step $n$. The small band region between the interface positions at time step $n$ and $n+1$ where no data exists is called the ghost region. This occurs due to the Langrangian style of approach, where the interface is tracked through time and points are displaced along with it. ALE methods could be used to discretise the equations of motion, so that the ghost node issues are alleviated, however, a Lagrangian approach is preferred here to provide greater accuracy in both the solution and multi-zone matching conditions.

In the multi-zone representation, transport data such as the fluid velocity and temperature are only known in the specified zones at a specified time step, and do not exist outside of these regions, meaning the zone 1 fluid velocity (and hence PDE value) at time step $n$ cannot be reconstructed accurately by the numerical method in zone 2. Extrapolation schemes can be used to reconstruct the data needed from the previous time step in a small band outside of the interior zone [34], however, this limits the convergence and accuracy of the numerical method to that of the extrapolation scheme, which can be very poor when the data varies sharply over the interface.

The auxiliary method alleviates extrapolation by not having PDE points placed on the interface, and using a local enforcement of the matching condition in order that global accuracy is still maintained. Internal PDE points that fall in the ghost region as the interface moves are simply translated into the inner region, which is possible due to the meshless nature of the scheme. The auxiliary multi-zone representation is much more in keeping with the original boundary value style of the RBF-FC method than the embedded representation, and allows the meshless RBF-FC nodes to evolve with the interface whilst accurately enforcing the matching conditions.

We utilise a coupled numerical scheme here, as opposed to solving the entire problem solely with boundary elements, or radial basis functions, in order that the accuracy and efficiency of the scheme are maximized. When using solely boundary elements, some form of dual reciprocity method would have to implemented to evaluate the perturbed pressure components (effectively solving a Poisson equation), which would require some form of RBF interpolation. The heat transfer equation would also involve domain integrals when solved with the BEM, making the scheme inefficient for the large scale problems tackled here. In a similar vein, the RBF-FC method is not as accurate (or efficient) for the solution of the homogeneous pressure as the BEM, making it less desirable when solving the entire problem. 


\section{Coupled pressure/flow - Heat Transfer Algorithm}

We solve the coupled thermo-viscous problem using the numerical methods described in the previous section following the algorithm below:

1. Set-up the initial interface using 8 finger symmetric perturbation, $r=1+\epsilon_{0} \cos (8 \theta)$. Setup the initial temperature field using a smoothed step function over the interface using equation (1) with $\delta=1$. Generate the boundary element mesh and embedded RBF-FC nodal arrangement.

2. Use the initial temperature field to calculate the viscosity using (5). Solve the initial pressure field using the initial interface position with equations (12) and (13), to calculate the the total velocity with (21).

3. Displace the interface using a forward Euler time step, i.e $\Delta x_{i}=n_{i} U_{n} \Delta t$. Advance $t=t+\Delta t$.

4. Generate (on the first time step) or update the auxiliary/embedded RBF-FC nodes using the quadtree dataset at the new interface position. Displace relevant nodes onto the interface. Generate heat transfer PDE data at the new interface location, using the old time step data (simply interpolate initial data for the first time step). This involves reconstructing $\bar{S}_{l}(x, t)$ at the current time step for every internal RBF node, using the previous time step RBF local systems (relevant local stencils are chosen for new/displaced nodes based on their corresponding locations). See appendix for more details.

5. Solve the transient heat transfer equation (22) using the auxiliary RBF-FC method.

6. Use the temperature field at the new interface location to calculate the viscosity everywhere using (5).

7. Solve the pressure field using the new viscosity field and interface with equations (12) and (13), to calculate the the total velocity with (21).

8. Repeat steps $3-7$ until the end simulation time is reached.

\section{Fluid properties}

Before proceeding to the main body of the thermo-viscous fingering verification and results, we briefly describe the fluid properties and temperature regimes that will be used in these sections, summarised in Tables 1 and 2 below. We use the temperature regimes in Table 2 to correspond to those in real $\mathrm{CO}_{2}$ injection scenarios discussed in the introduction. Larger temperatures ranges could be used, in which the thermo-viscous effects would be correspondingly magnified.

We use $\mathrm{CO}_{2}$ viscosity data interpolated at the specified temperatures at $20 \mathrm{MPa}$ injection pressure from [35]. The brine viscosity is calculated from sodium chloride solution data at the specified temperatures at $0.5 \mathrm{Mol} / \mathrm{Kg}$ and $10 \mathrm{MPa}$ resident pressure using [36]. We use a suitable brine $-\mathrm{CO}_{2}$ surface tension of $0.03 \mathrm{~kg} / \mathrm{s}^{2}$ at the resident temperature and pressure values [37]. Due to the lack of data concerning thermal diffusivity values for brine, we use a corresponding diffusivity value for water at the resident temperature and pressure as $0.0015 \mathrm{~cm}^{2} / \mathrm{s}$, for supercritical $C_{2} O_{2}$ we take the diffusivity as $0.0005 \mathrm{~cm}^{2} / \mathrm{s}[38]$.

\begin{tabular}{|c|c|c|c|}
\hline$T\left({ }^{\circ} \mathrm{C}\right)$ & $\begin{array}{c}C O_{2} \text { viscosity } \\
\bar{\mu}_{1}(\text { Pa.s })\end{array}$ & $\begin{array}{c}\text { Brine viscosity } \\
\bar{\mu}_{2} \text { (Pa.s) }\end{array}$ & $\beta$ \\
\hline 35 & $8.411 \times 10^{-5}$ & $7.542 \times 10^{-4}$ & 8.967 \\
\hline 40 & $7.858 \times 10^{-5}$ & $6.865 \times 10^{-4}$ & 8.736 \\
\hline 45 & $7.376 \times 10^{-5}$ & $6.283 \times 10^{-4}$ & 8.519 \\
\hline 50 & $6.893 \times 10^{-5}$ & $5.779 \times 10^{-4}$ & 8.384 \\
\hline 55 & $6.456 \times 10^{-5}$ & $5.338 \times 10^{-4}$ & 8.268 \\
\hline 60 & $6.019 \times 10^{-5}$ & $4.952 \times 10^{-4}$ & 8.227 \\
\hline 65 & $5.664 \times 10^{-5}$ & $4.610 \times 10^{-4}$ & 8.139 \\
\hline 70 & $5.309 \times 10^{-5}$ & $4.306 \times 10^{-4}$ & 8.112 \\
\hline
\end{tabular}

Table 1: $\mathrm{CO}_{2}$ and Brine fluid properties with temperature.

The injection regimes in Table 2 summarise the mobility values for each specific injection temperature and the associated rate of change of viscosity with temperature for the specific regime. 
The values of $\alpha$ change, since the non-dimensional scalings change with the resident temperature, meaning that $\alpha$ must be varied to keep the same dimensional dependence on temperature. The plate separation $b$ is chosen as $0.00146731 \mathrm{~cm}$, meaning the intrinsic permeable of the cell is $1.8 \times 10^{-11} \mathrm{~cm}^{2}$ (equivalent to oil reservoir rock).

\begin{tabular}{|c|c|c|c|c|c|c|c|}
\hline Case & $T_{0}\left({ }^{\circ} \mathrm{C}\right)$ & $T_{\infty}\left({ }^{\circ} \mathrm{C}\right)$ & $\bar{M}_{1}\left(\mathrm{~m}^{3} \cdot \mathrm{s} / \mathrm{kg}\right)$ & $\bar{M}_{2}\left(\mathrm{~m}^{3} \cdot \mathrm{s} / \mathrm{kg}\right)$ & $\beta$ & $\alpha_{1}$ & $\alpha_{2}$ \\
\hline 1 & 35 & 70 & $2.133 \times 10^{-7}$ & $2.379 \times 10^{-8}$ & 8.967 & 0.4583 & 0.5390 \\
\hline 2 & 35 & 50 & $2.133 \times 10^{-7}$ & $2.379 \times 10^{-8}$ & 8.967 & 0.1986 & 0.2627 \\
\hline 3 & 35 & 35 & $2.133 \times 10^{-7}$ & $2.379 \times 10^{-8}$ & 8.967 & 0.0000 & 0.0000 \\
\hline 4 & 50 & 35 & $2.603 \times 10^{-7}$ & $3.105 \times 10^{-7}$ & 8.384 & 0.1986 & 0.2627 \\
\hline 5 & 70 & 35 & $3.380 \times 10^{-7}$ & $4.167 \times 10^{-7}$ & 8.112 & 0.4583 & 0.5390 \\
\hline
\end{tabular}

Table 2: Summary of injection regimes and fluid properties

\section{Mass conservation validation}

In this section, we demonstrate the fully coupled numerical scheme on the case of the displacement of an initially circular interface, with zero perturbation. Perturbations will only grow after a long period of time due to numerical error, meaning that the interface should propagate as a growing circle with an increasing volume equal to that injected at the origin. By comparing the numerical volume of the growing plume and the analytical volume injected at the origin, we can validate that the coupled scheme is mass conservative and that the governing equation of the flow (2) is being satisfied. The total volume of fluid is given in non-dimensional form as:

$$
V_{a}=V_{0}+\bar{b} t
$$

Where, $V_{0}$ is the initial volume of fluid in the cell. If the interface displaces as a circle with radius $r(t)$ in a uniform cell, the volume can be expressed as:

$$
\begin{aligned}
V_{a}=\pi \bar{b} r(t)^{2} & =\bar{b}(\pi+t) \\
r(t) & =\sqrt{\frac{\pi+t}{\pi}}
\end{aligned}
$$

To ensure that mass has been conserved in the numerical method, the volume of fluid can be calculated through numerical integration of the evolving interface (using the average interface position $\bar{r}$ ) and compared to the value given by equation (27). The BEM has been validated for homogeneous mobility cases in previous work by [30], meaning the mass conservation tests presented here will validate that the RBF-FC method is contributing a correct velocity (from the perturbed pressure) to displace the interface and that the corresponding temperature field transports accurately. The RBF method still solves the full multi-zone steady-state pressure and the transient heat transfer problem, but with the interface given by a simple circle instead of the convoluted interface usually found in viscous fingering problems.

Given the symmetry of the problem, we use a triangular solution domain for the RBF-FC methods, similar to that shown in Figure 2. We use two different nodal distributions for the perturbed pressure solution and the transient heat transfer solution. This ensures numerical accuracy and stability for both solutions, which do not necessarily need the same nodal distribution. As the temperature profile is sharper than the perturbed pressure, the outer boundary can be brought closer to the injection source and the number of nodal points increased in the domain. Also, as the resulting global matrix for transient problem is sparsely populated, increasing the number of nodes does not significantly increase the solution cost.

For the heat transfer we use an outer boundary at $x_{1}=8$, with a maximum quadtree level of 8 , and minimum 4. For the perturbed pressure we use an outer boundary at $x_{1}=32$, to ensure that the perturbed pressure has dropped to near zero in the far field where the boundary condition is applied. The temperature (needed for the viscosity) at any point $x>8$ is taken at the outer boundary value $T_{\infty}$. The maximum quadtree level for the perturbed pressure is set at 10 , with a 
minimum of 3. This ensures that both RBF nodal distributions cluster points around the interface with a minimum nodal separation of $\Delta x=0.03125$. The transient heat transfer problem uses a much denser distribution of nodal points, with the $B_{t}$ value (defining the sharpness of the distance based splitting criteria, see [29]) varying uniformly from 11 at the highest cell level to 6 at the lowest cell level. We also ensure that the nodes around the injection source are at the highest cell level, stability. This constant, fine clustering of nodes extends to a point $r=3$ in the temperature domain. With the perturbed pressure nodes, we vary $B_{t}$ uniformly from 3 at the highest level, to 6 at the lowest cell level, creating a much coarser dataset that can still accurately capture the perturbed pressure.

\begin{tabular}{|c|c|c|c|c|}
\hline \multirow{2}{*}{$\begin{array}{c}\text { Injection } \\
\text { Regime }\end{array}$} & \multicolumn{2}{|c|}{ Relative Error, $Q=0.5 \mathrm{~cm}^{2} / \mathrm{s}$} & \multicolumn{2}{c|}{ Relative Error, $Q=0.75 \mathrm{~cm}^{2} / \mathrm{s}$} \\
\cline { 2 - 5 } & $\Delta t=0.02$ & $\Delta t=0.04$ & $\Delta t=0.02$ & $\Delta t=0.04$ \\
\hline $35^{\circ} \mathrm{C}-50^{\circ} \mathrm{C}$ & $7.27 \times 10^{-4}$ & $1.47 \times 10^{-3}$ & $7.70 \times 10^{-4}$ & $1.44 \times 10^{-3}$ \\
\hline $50^{\circ} \mathrm{C}-35^{\circ} \mathrm{C}$ & $7.02 \times 10^{-4}$ & $1.39 \times 10^{-3}$ & $6.00 \times 10^{-4}$ & $1.55 \times 10^{-3}$ \\
\hline $35^{\circ} \mathrm{C}-70^{\circ} \mathrm{C}$ & $2.34 \times 10^{-3}$ & $2.32 \times 10^{-3}$ & $2.57 \times 10^{-3}$ & $3.16 \times 10^{-3}$ \\
\hline $70^{\circ} \mathrm{C}-35^{\circ} \mathrm{C}$ & $1.86 \times 10^{-4}$ & $7.01 \times 10^{-4}$ & $6.38 \times 10^{-4}$ & $7.30 \times 10^{-4}$ \\
\hline
\end{tabular}

Table 3: Relative errors between analytical and numerical volume of fluid for different injection regimes at $t=10$.

We maintain a constant non-dimensional shape parameter value of $c^{*}=90$ for all simulations. In the boundary element method the full interface is solved, around which a target element size is maintained throughout the simulations at $\Delta x \approx 0.06$, ensuring a mesh-independent boundary element solution. Both RBF nodal distributions are therefore twice as refined around the interface as the BEM. These nodal distributions have been thoroughly tested, and the cell levels increased by two levels beyond those quoted here, creating an 8 fold increase in the nodal density compared to the BEM. At these higher levels, no further gain in accuracy is achieved, without significantly reducing the time step size to impractically small values.

In Table 3, the relative errors between the analytical and numerical volume of fluid are shown for different temperature regimes and time step sizes. All cases exhibit very low relative errors, with the largest error coming from the $35^{\circ} \mathrm{C}-70^{\circ} \mathrm{C}$ case which has the largest local capillary numbers. At the lower temperature injection we see $1^{\text {st }}$ order convergence in the time stepping scheme as expected, however, in the high injection cases the error plateaus. This is due to the solution accuracy reaching the limit imposed by the spatial discretisation, so no further reduction in time step increases the accuracy (at least when $Q$ is high). We use the time step size of $\Delta t=0.02$ for all the following numerical results to ensure numerical accuracy and stability.

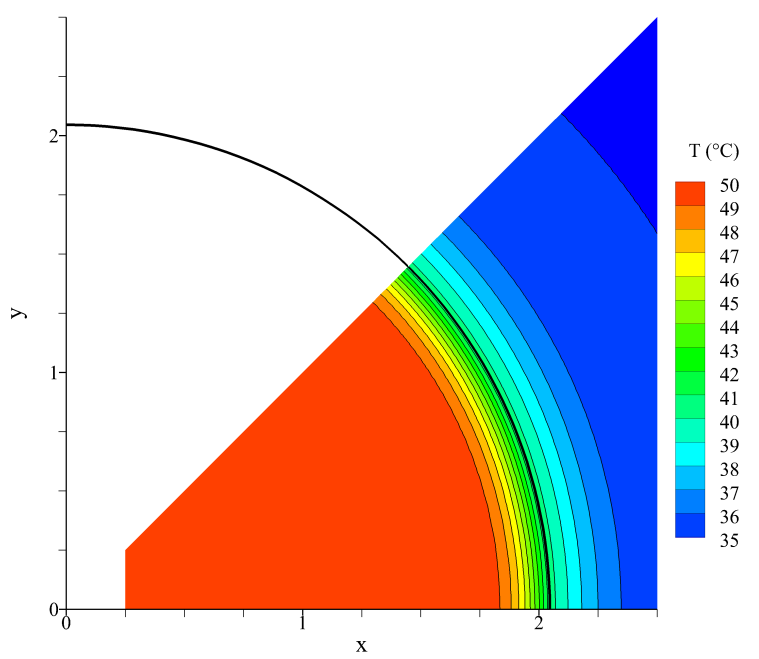

Figure 3: Temperature contour and interface plot at $\mathrm{t}=10$ for the $50^{\circ} \mathrm{C}-35^{\circ} \mathrm{C}, Q=0.5 \mathrm{~cm}^{2} / \mathrm{s}$ case. The bold black interface overlays a temperature contour plot with thin contour lines of the temperature field.

Figure 3 shows the interfacial displacement and temperature field for the $50^{\circ} \mathrm{C}-35^{\circ} \mathrm{C}$ case with 
$Q=0.5 \mathrm{~cm}^{2} / \mathrm{s}$ at $t=10$. This highlights the high Peclet number, as the temperate field shows very little diffusion and the interface lies at a temperature close to the initial temperature condition, i.e. the initial condition has been almost purely advected. The contour lines bunch in the inner region with the low diffusivity and high convection, showing the sharp drop in temperature at the interface that is then smoothly diffused in the outer region. The numerical solution still shows a highly circular interface, indicating that numerical error has not yet caused any perturbations to grow. To maintain numerical stability with the numerical methods, a combination of smoothing algorithms are used that damp severe numerical oscillations. Brief summaries of these techniques are given below.

\subsection{Artificial diffusion and smoothing}

To ensure stability with the $2^{\text {nd }}$ order Crank-Nicholson scheme $(\theta=0.5)$ for the auxiliary RBFFC heat transfer solution, artificial diffusion and selective smoothing are employed. In situations when the heat transfer is highly convective (i.e. when the thermal Peclet number is very high), the solution can sometimes briefly oscillate above/below the base values of the injection and resident temperatures, bringing numerical error into the solution. This occurs due to the relative instability of the RBF solution when high shape parameters are used, and the $2^{\text {nd }}$ order time stepping scheme which is conditionally stable. These oscillations only occur for a very short amount of time in localised regions, but if unchecked can propagate and eventually destroy the solution.

When the solution exceeds a threshold based on the injection or resident temperature (typically $0.1 \%$ above/below), an artificially high diffusion is applied to the point for a single time step that is 100 times higher than the base diffusivity. This damps any severe numerical oscillations, whilst ensuring that there is negligible effect on the solution profile. The threshold for the artificial diffusion and selective smoothing (detailed below) are chosen empirically from numerical testing across a range of values. Some simulations (particular cold injection regimes, which are relatively more stable), require no additional stabilisation techniques. The effect of the stabilisation on the resulting solution accuracy was tested, and deemed negligible, by comparing the solutions to those obtained without stabilisation at higher spatial and temporal resolutions.

The artificial diffusion here acts differently to the traditional artificial viscosity that is used in damping oscillations occurring in shock wave propagation. Artificial viscosity is added into the momentum equations to smooth small oscillations, but the artificial diffusivity used in this work only affects the transport equation for the temperature. The velocity and pressure fields remain unaffected by this process.

We use a $2^{\text {nd }}$ order Crank-Nicholson time stepping scheme $(\theta=0.5)$, as opposed to a fully implicit $(\theta=1)$ or forward Euler $(\theta=0)$ scheme to maximize solution accuracy. In testing, the fully implicit (which does not necessarily require artificial diffusion, due to unconditional stability) and forward Euler schemes were much less accurate globally and required impractically small time step sizes to achieve an acceptable solution accuracy.

Selective smoothing is employed, whereby if a solution point differs from the average of its neighbours by a certain tolerance (we set this to a high value usually $\approx 7.5 \%$ ), then it is smoothed to the average value of its neighbours. Artificial diffusion and selective smoothing occur very infrequently in the simulations due to the highly refined datasets, generally being applied to around 1-5 points every hundred time steps. This is enough to maintain numerical stability and allow the simulations to run for very long periods of time without affecting the solution accuracy. For example, in the $70^{\circ} \mathrm{C}-35^{\circ} \mathrm{C}$ case in Table 3 with $\Delta t=0.02$, the percentage of total points (over the whole simulation time) where artificial diffusion and selective smoothing are applied is $8.2 \mathrm{x}$ $10^{-4} \%$ and $8.9 \times 10^{-3} \%$ respectively, indicating the sparse application of numerical smoothing.

\subsection{Shape parameter relaxation}

For the embedded RBF-FC pressure/velocity solution, we employ shape parameter relaxation to ensure smooth variation in the pressure/velocity field between time steps. We maximise the initial shape parameter, using the highest value that can be achieved whilst still allowing the local systems to be solved using quad precision arithmetic. This is done on a problem specific basis, by raising the shape parameter until the matrices become to ill-conditioned to be readily inverted. A single value of the non-dimensional shape parameter is used, which when scaled to the 
node separation means the dimensional shape parameter increases with distance away from the interface.

For the relaxation scheme, we set the shape parameter initially high at each time step as $c^{*}=90$, and evaluate the residual change in interfacial velocity compared to the previous time step. If the change between time steps is over $5 \%$ we drop the shape parameter by $10 \%$ successively relaxing the solution (up to 30\%) until the residual change is less than $5 \%$. Since the nodal discretisation changes with each time step, some datasets show a more regular distribution around the interface (depending on its shape) and hence perform better. For poorer quality datasets, relaxing the shape parameter helps to ensure stability whilst maintaining solution accuracy and a smoothly varying velocity field. This process also occurs very infrequently, occurring on the order of 10 times in the whole simulation (of over 1500 time steps).

\section{Thermo-viscous fingering results}

We now consider the cases of non-isothermal displacement given in Table 2 with an initial interface perturbation. All cases are run with the mesh/time step parameters discussed in the previous section. An isothermal case at $35^{\circ} \mathrm{C}$ is used as the base case to compare non-isothermal results with, since this represents the common temperature when raising the injection temperature for hot injection cases and raising the resident temperature for cold injection cases. We break the results section up into subsections detailing the different characteristics of the flow, starting with the general system behaviour below.

\subsection{General system behaviour}

Here, we describe the general system behaviour using interfacial plots and timeseries data of the key parameters. In Figure 4 , the interfacial displacements at $t=30$ can be seen for the different temperature regimes at two different injection fluxes, $Q=0.5 \mathrm{~cm}^{2} / \mathrm{s}$ and $Q=0.75 \mathrm{~cm}^{2} / \mathrm{s}$. These two injection fluxes will be used throughout to vary the effective capillary number.

The lower injection flux regimes, plotted in Figures 4(a) and (b) illustrate the effect of a nonisothermal temperature distribution in the domain. In the cold injection cases in Figure 4(a), the interfaces have been stabilised in comparison to the isothermal; the finger bases are more perturbed, whilst the fronts remain closer to the injection source, creating a less ramified structure. Increasing the resident temperature from $35^{\circ} \mathrm{C}$ to $70^{\circ} \mathrm{C}$ has significantly stabilised the interface, delaying the onset of bifurcation.

In corresponding hot injection cases in Figure 4(b), the interfaces have been destabilised, with the fronts accelerated beyond the isothermal case. The larger temperature difference case of $70^{\circ} \mathrm{C}-$ $35^{\circ}$ has produced a more significant destabilisation, resulting in an earlier bifurcation and more perturbed finger front at $t=30$ in $4(\mathrm{~b})$. Interestingly, the bases remain largely in the same position, indicating that the base position is controlled mainly by the outer fluid temperature.

Increasing the injection flux to $Q=0.75 \mathrm{~cm}^{2} / \mathrm{s}$ in Figure $4(\mathrm{c})$ and (d) results in more significant (de)stabilisation effects from the non-isothermal regimes due to the increased capillary number. In the cold non-isothermal cases, the interfaces are somewhat stabilised in Figure 4(c), with the bases all displaced further than the isothermal case. However the finger fronts now show different bifurcation regimes to the isothermal, due to the raised capillary number and increased thermal effects. Generally, raising the resident temperature has stabilised the front, with the $35^{\circ} \mathrm{C}-70^{\circ} \mathrm{C}$ case clearly showing a less advanced finger front than the isothermal case.

For the hot injection cases at the higher flux of $Q=0.75 \mathrm{~cm}^{2} / \mathrm{s}$ in Figures $4(\mathrm{~d})$, the interfaces have been accelerated and show elongated two finger split regimes. The two finger regime usually represents a more stable bifurcation than the three finger split, as viscous forces are lower in comparison to surface tension forces. However, in this case, the two finger split is caused by a different mechanism than in isothermal viscous fingering bifurcations, discussed in more detail in section 7.2 .

The displacement regimes can be analysed quantitatively using time series data of the finger growth rate, pressure gradient and mobility, shown in Figure 5. The dimensional pressure gradient and mobility are shown here (plotted against non-dimensional time), as opposed to the dimensionless quantities since the homogeneous mobility $\bar{M}_{2}$ (and hence scaling) changes with the resident temperature. This makes making comparison between hot and cold injection regimes difficult, with 


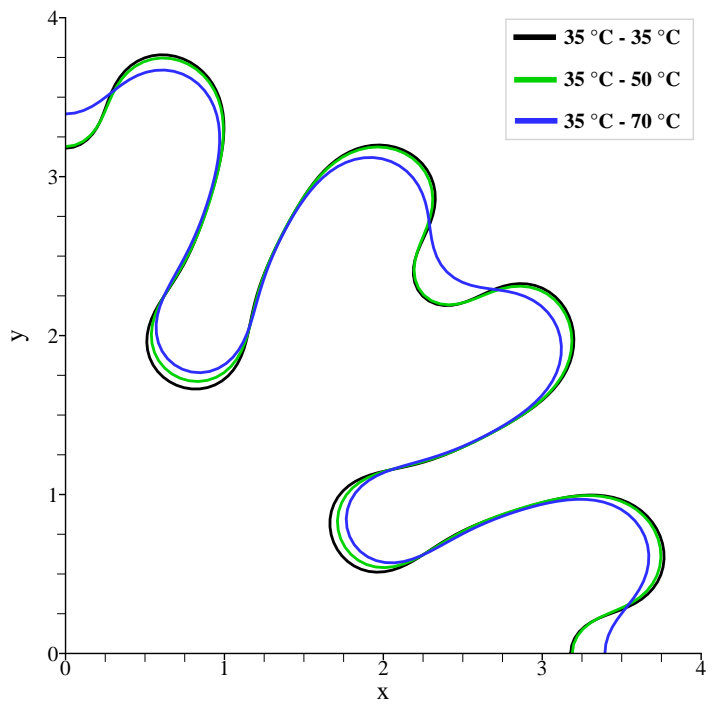

(a) $Q=0.5 \mathrm{~cm}^{2} / \mathrm{s}$

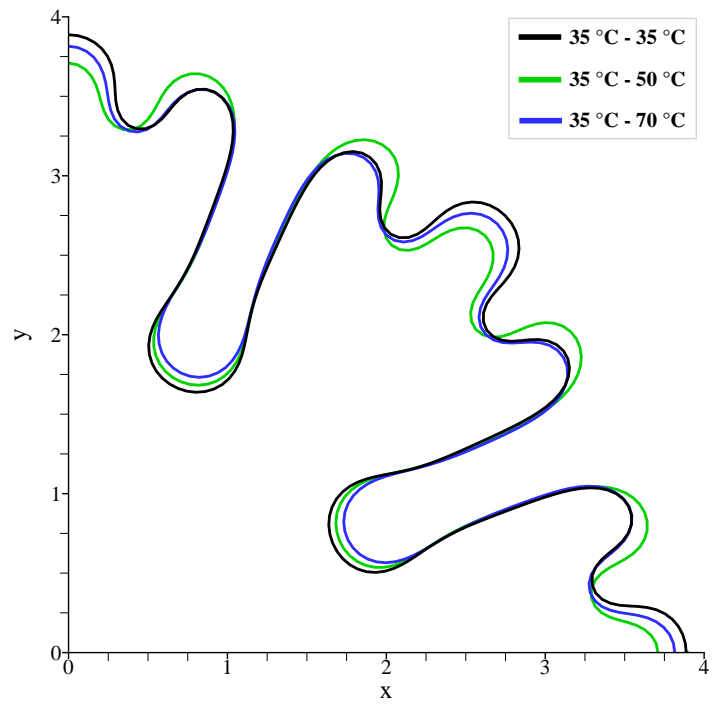

(c) $Q=0.75 \mathrm{~cm}^{2} / \mathrm{s}$

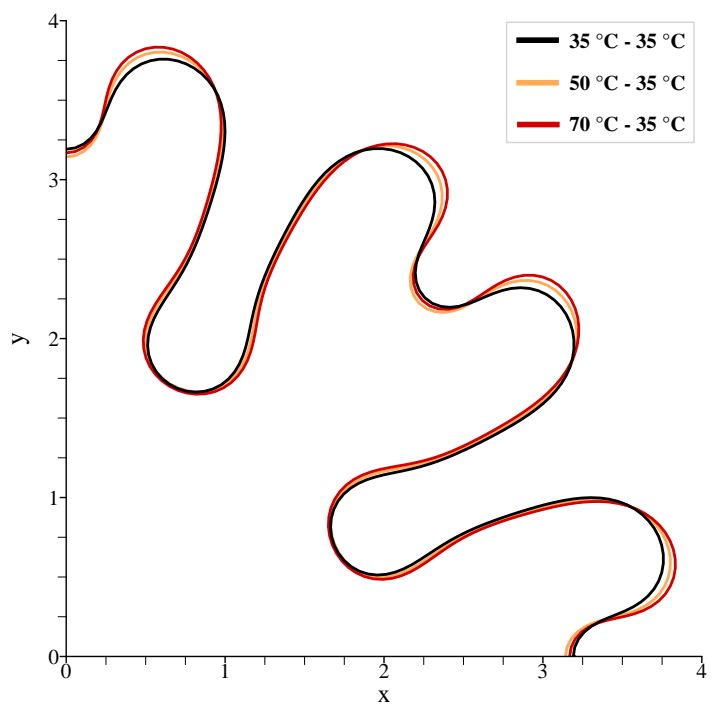

(b) $Q=0.5 \mathrm{~cm}^{2} / \mathrm{s}$

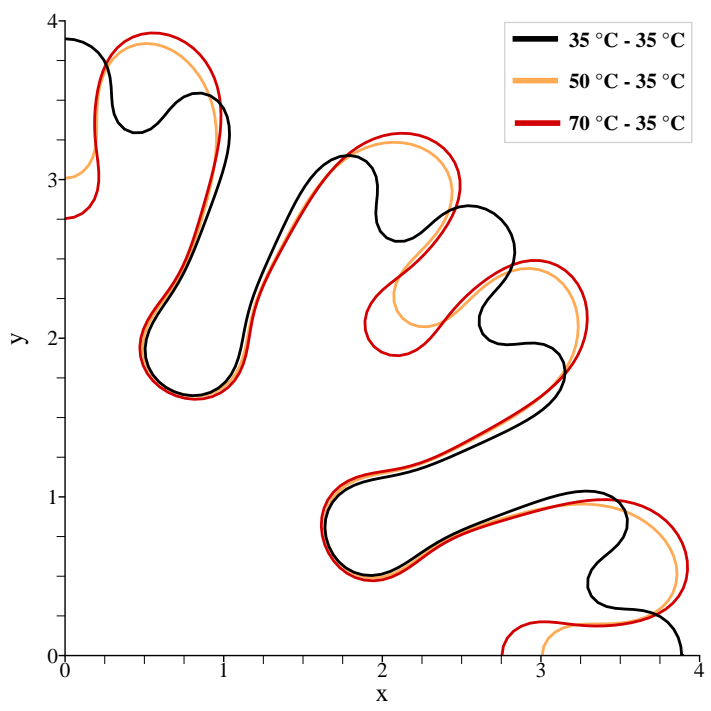

(d) $Q=0.75 \mathrm{~cm}^{2} / \mathrm{s}$

Figure 4: Interface plots at $t=30$ for several non-isothermal injection regimes; (a) and (c) show cold injection cases, (b) and (d) show hot injection cases.

the dimensional values giving a better absolute indication of the relative impact of thermal effects. Since the growth rate and time are unaffected by the temperature regime, these are presented in non-dimensional form. The dimensionless non-linear growth rate of a perturbation with radial position $r$ is given by:

$$
\sigma_{t}=U_{n}(r, t)-U_{n 0}(r, t)
$$

Where, $U_{n}(r, t)$ is the normal velocity at the perturbed interface and $U_{n 0}(r, t)$ is the normal velocity at the unperturbed interface (growing in an isothermal regime). The velocity of the unperturbed interface with time is given purely by the source injection velocity at the corresponding radius given by $r(t)=\sqrt{\frac{\pi+t}{\pi}}$.

The growth rate shown in Figure 5(a) highlights the initial acceleration of the finger fronts due to the high temperature injection and the initial deceleration of the front due to the low temperature injection. The growth rate is controlled by the evolution of the mobility and pressure gradient at the interface, shown in Figure 5(c) - (f). The initial difference in growth rate between the cases is due to the change in pressure gradient, as the hot and cold injection cases have the 


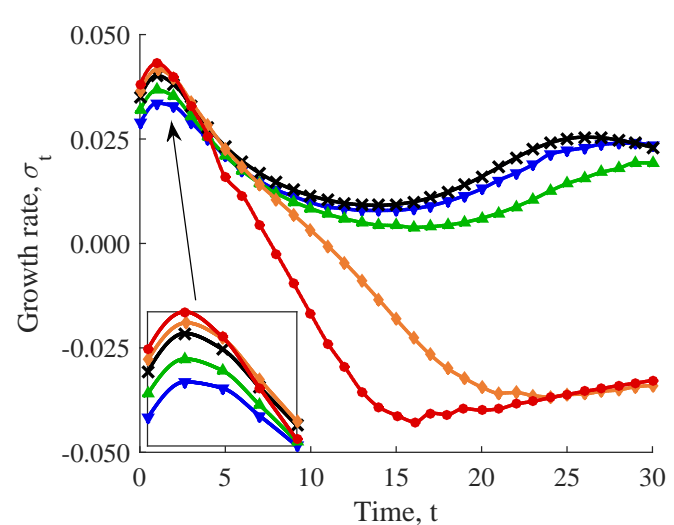

(a)

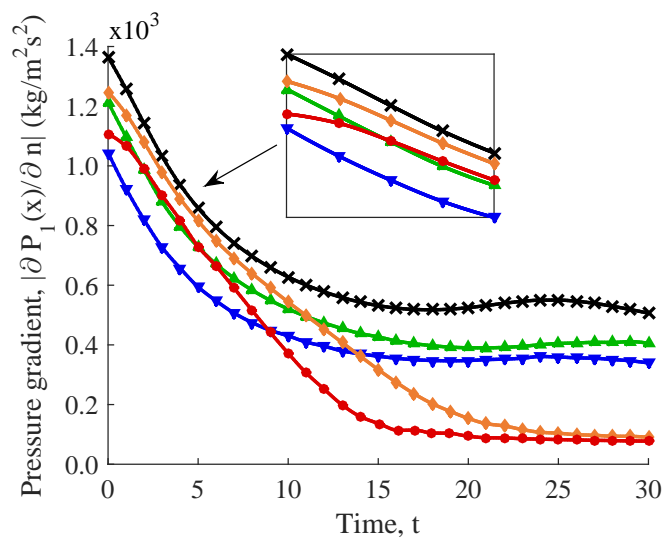

(c)

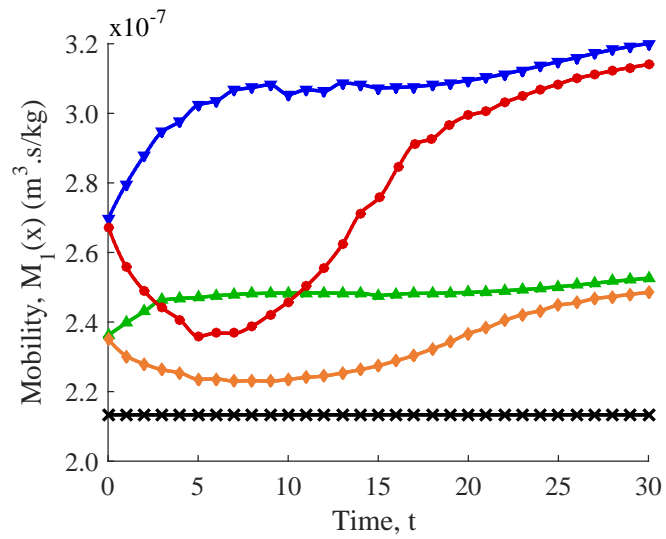

(e)

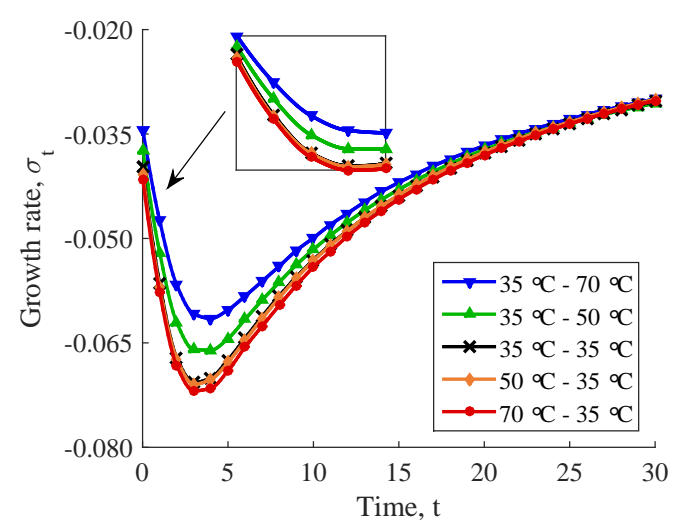

(b)

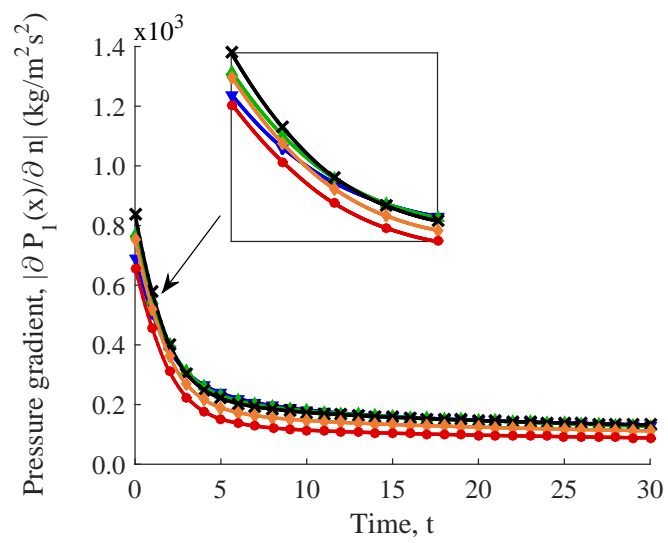

(d)

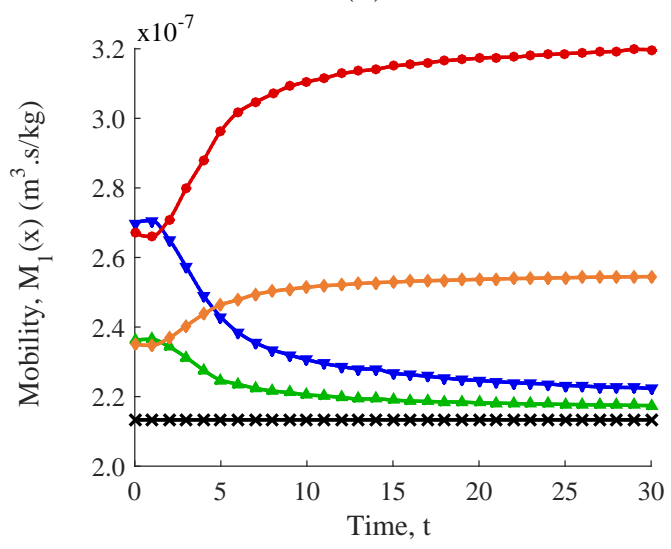

(f)

Figure 5: Plots of the growth rate, pressure gradient and mobility with time for the finger fronts (a, c, e) and finger bases $\left(\mathrm{b}, \mathrm{d}, \mathrm{f}\right.$ ) for different temperature regimes with $Q=0.75 \mathrm{~cm}^{2} / \mathrm{s}$. Legend for each plot shown in (b)

same mobilities for the same initial temperatures (i.e. the $35^{\circ} \mathrm{C}-70^{\circ} \mathrm{C}$ case has the same initial temperature and mobility at the interface as the $70^{\circ} \mathrm{C}-35^{\circ} \mathrm{C}$ case).

In cold injection cases, the pressure gradient is significantly reduced from the isothermal (see Figure 5(c)), as the perturbed pressure gradient component in equation (21) is positive, which reduces the velocity and hence growth rate. The magnitude of the total pressure gradient is initially reduced by $23.7 \%$ for the $35^{\circ} \mathrm{C}-70^{\circ} \mathrm{C}$ case compared to the isothermal, whereas the mobility is raised by only $20.9 \%$, which results in the lower growth rate of the $35^{\circ} \mathrm{C}-70^{\circ} \mathrm{C}$ case. The reverse is true of the hot injection case, where a negative perturbed pressure gradient causes a reduction in magnitude of the total pressure gradient by only $19.0 \%$ for the $70^{\circ} \mathrm{C}-35^{\circ} \mathrm{C}$ case, 
with the mobility increasing by $20.9 \%$, resulting in a higher growth rate.

At the finger bases, the growth rate behaviour is reversed, i.e. the cold cases have higher base growth rates than the hot cases (see Figure 5(b)). However, the mechanism for the increase/decrease in growth rate is the same as at the fronts. The cold injection cases have a higher growth rate at the finger bases, due to the increased pressure gradient (note the perturbed pressure gradient in equation (21) is negative at the base, increasing the velocity). With time, the base growth rates all tend to the same value, when the mobility an pressure gradient profiles have both reached near constant values. At this stage, the temperature fields are diffuse and the increase/decrease in pressure gradient due to the cold/hot injection is equalled by the decrease/increase of the temperature and mobility at the base, resulting in the same growth rate.

The negative growth rates that occur in Figure 5(a) and (b) are caused by bifurcations. At bifurcated points of the interface where the curvature is negative (for instance at the finger bases), the normal velocity is reduced significantly in comparison to an unperturbed interface, meaning the growth rate given by equation (30) becomes negative.

The pressure gradient represents the primary control for the initial growth of the interface, when the temperature field evolves in a highly convective regime (i.e. the time frames highlighted by the zoomed in plots in Figure 5). If the temperature field grew in a purely convective manner, this regime would hold for all time, with hot injection cases being relatively more unstable than isothermal and cold injection cases. However, with diffusion in the physical system, the temperature field evolves with different profiles around the interface for the hot and cold injection cases that alter the bifurcation mode. It is worth noting that in the lower injection flux regime at $Q=0.5$ $\mathrm{cm}^{2} / \mathrm{s}$, the same effects occur as detailed above but with reduced magnitude, resulting in the early timeframe acceleration/deceleration of the interface, but without alteration in the bifurcation mode.

The promotion of finger bifurcation in the hot injection cases here is similar to that reported experimentally by [22], albeit in the miscible regime and at slightly higher injection fluxes than those presented here. In a small range of mobility ratios $\beta \approx 10$, they find that bifurcation occurs earlier in the evolution for the hot non-isothermal regime. These results appear at least qualitatively very similar to those here, whereby the finger fronts are accelerated in hot injection regimes.

\subsection{Thermal evolution $\&$ bifurcation modes}

After the initial growth at $t=5$ in the $Q=0.75 \mathrm{~cm}^{2} / \mathrm{s}$ regime, the growth rate of the two hot injection regimes in Figure 5(a) starts to vary significantly from the cold injection and isothermal cases as the bifurcation changes to a two finger mode. The difference in growth rate and bifurcation mode can be explained by considering the temperature and mobility evolution. In Figure 5(e) the front mobility (and temperature) drops significantly when $0<t<5$ in the hot injection cases, as the temperature is rapidly convected towards the finger tip and diffused into the surrounding fluid.

As the tip mobility drops, the base mobility rises in the hot cases in Figure 5(f). As the base develops a high negative curvature as time progresses, heat is diffused from the base and sides of the finger into the spacing between the fingers, creating a high temperature region. This raises the temperature of the base until it reaches a near constant value where the base convection matches the diffusion into the outer region. This difference in temperature evolution between the finger fronts and base results in the difference between the bifurcation regimes for the hot and cold injection cases at the high injection flux.

The temperature profile around the interface for the hot and cold injection regimes at $t=5$ in Figure 6 highlights the cause of the different bifurcation modes between the hot and cold cases with $Q=0.75 \mathrm{~cm}^{2} / \mathrm{s}$. In the cold injection case in Figure 6(a), the temperature increases monotonically from the finger base to the tip, meaning the highest mobility (and hence velocity) is found at the finger tip. The interface proceeds to sharpen in time, and form the three finger bifurcation.

However, in the hot injection case, the temperature decreases monotonically from the finger base to the tip, meaning the finger sides have a higher mobility than directly at the tip. This causes the velocity to be higher at the finger sides (highlighted by the red velocity vector in 6 (b)) and the spreading mechanism is enhanced. This creates a 'flatter' finger front and leads to the two finger bifurcation later in time. 


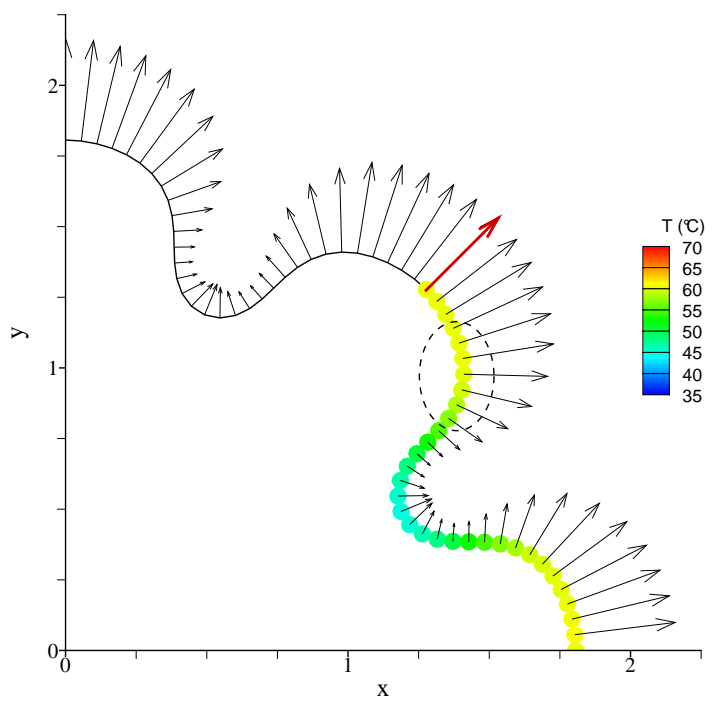

(a) $35^{\circ} \mathrm{C}-70^{\circ} \mathrm{C}$

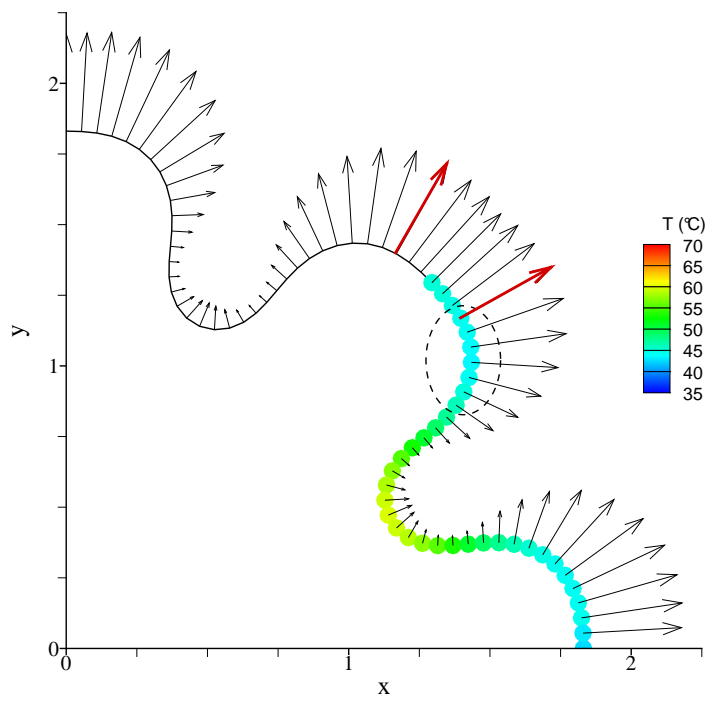

(b) $70^{\circ} \mathrm{C}-35^{\circ} \mathrm{C}$

Figure 6: Temperature scatter plots around the interface for the hot and cold injection cases at $t=5, Q=0.75$ $\mathrm{cm}^{2} / \mathrm{s}$. The arrows show normal velocity vectors $U_{n}$, with red arrows highlighting the maximum velocity.

At the stage shown Figure 6, diffusion effects are becoming more significant and have altered the temperature profile around the interface enough to cause the change in bifurcation regime. From this we can infer that the macroscopic temperature profile around the interface controls the late stage splitting mode of the interface, whilst the early time scale pressure gradient in the convective regime describes the general acceleration/deceleration effects of the hot/cold injection regimes.

Very similar macroscopic temperature profiles can also be seen later on in the finger growth at $t=30$ in Figure 7(a) and (b). Here, after the first bifurcation, the finger tips in the hot injection case have lower temperatures than the sides and base, indicating that the spreading mechanism is again being enhanced. Likewise, the cold injection cases have the highest temperature at the finger tips, showing that the fingers will be sharpened and will likely split into more fingers on bifurcation. The temperature contour plots at $t=30$ in Figure $7(\mathrm{c})$ and (d) show the convective nature of the flow, with the temperature profile closing following that of the interface. Here, the thermal Peclet number is very large at $P e=Q / D_{1}=1500$.

It is worth noting that we only see the two and three finger bifurcations here due to the capillary number and temperature regimes that are considered. For a four or five finger bifurcation, the interface has to become much more unstable, requiring a larger injection flux and capillary number $\left(C a_{g}>7000\right.$ produces a different bifurcation mode). However, the general mechanisms that we have presented here involving the bifurcation regime and acceleration/deceleration effects will still hold in these regimes, albeit with different variations in the bifurcation mode.

The temperature profiles across the finger front and base at $t=30$ can be seen in Figure 8. Here, the multi-zone nature of the temperature field is observable, where the gradient of the temperature profile decreases by a factor of three at the interface (highlighted by the crosses) due to the three fold increase in the diffusivity in the outer region $\left(D_{1}=0.0005 \mathrm{~cm}^{2} / \mathrm{s} D_{2}=0.0015 \mathrm{~cm}^{2} / \mathrm{s}\right)$. The temperature profile is very sharp in the inner region in the hot injection case as the front has bifurcated and formed a near stagnation point, and the heat transfer is dominated by diffusion. The front and base profile therefore exhibit very similar temperature profiles, with the base being slightly more diffuse since it was formed earlier in time. In the cold injection case, we still see the same change in temperature gradient at the interface, but the inner zone temperature field is much more diffuse as the front has yet to bifurcate and stagnate.

The viscosity profiles in Figure 8(c) and (d) show the relative increase/decrease in local mobility ratio at the interface for the cold/hot injection regimes. As the temperature increases towards the interface for the cold injection case, the mobility ratio is increased due to the relative change in 


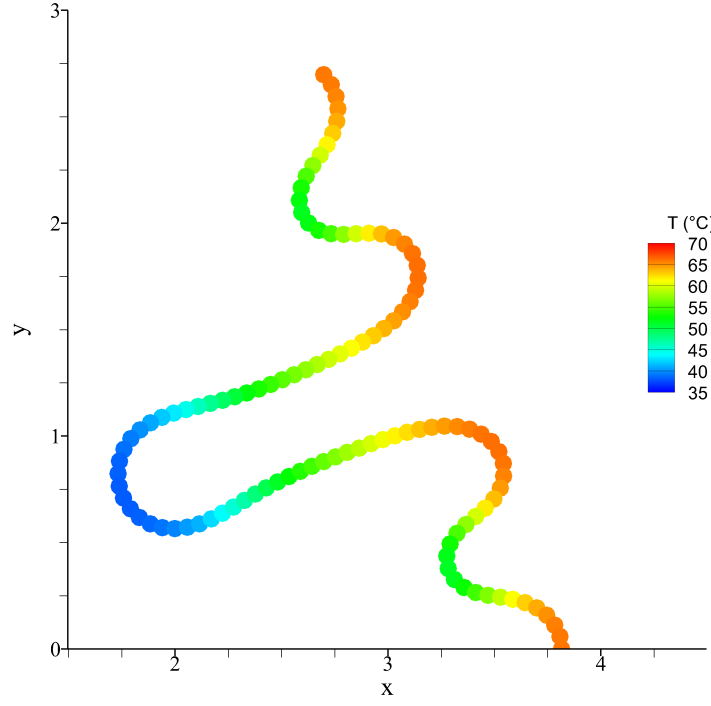

(a) $35^{\circ} \mathrm{C}-70^{\circ} \mathrm{C}$

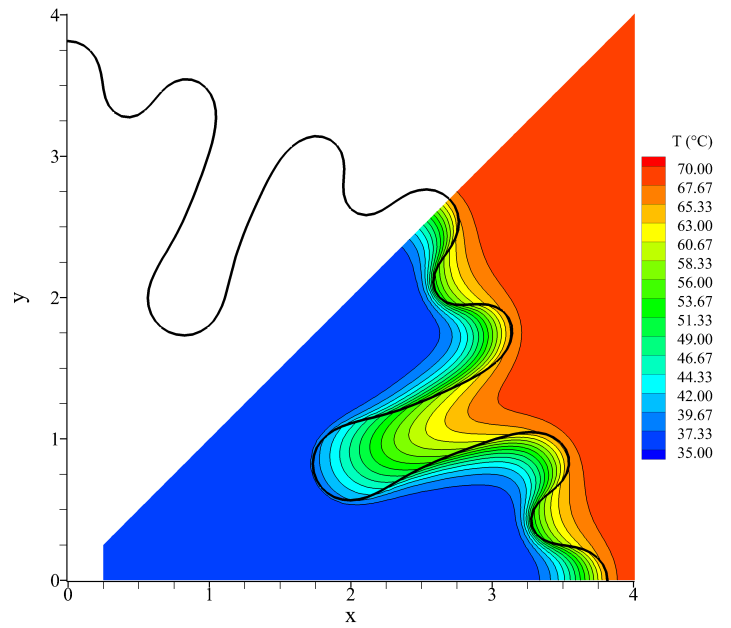

(c) $35^{\circ} \mathrm{C}-70^{\circ} \mathrm{C}$

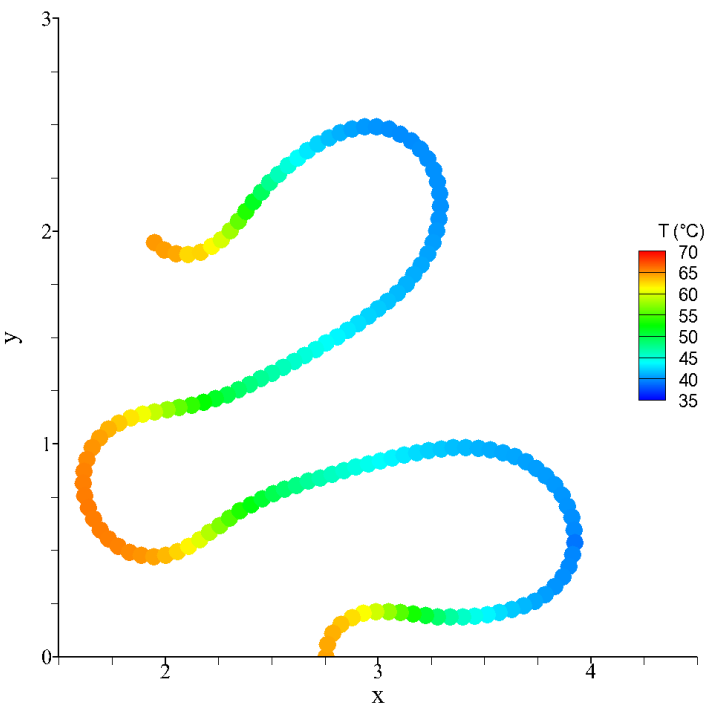

(b) $70^{\circ} \mathrm{C}-35^{\circ} \mathrm{C}$

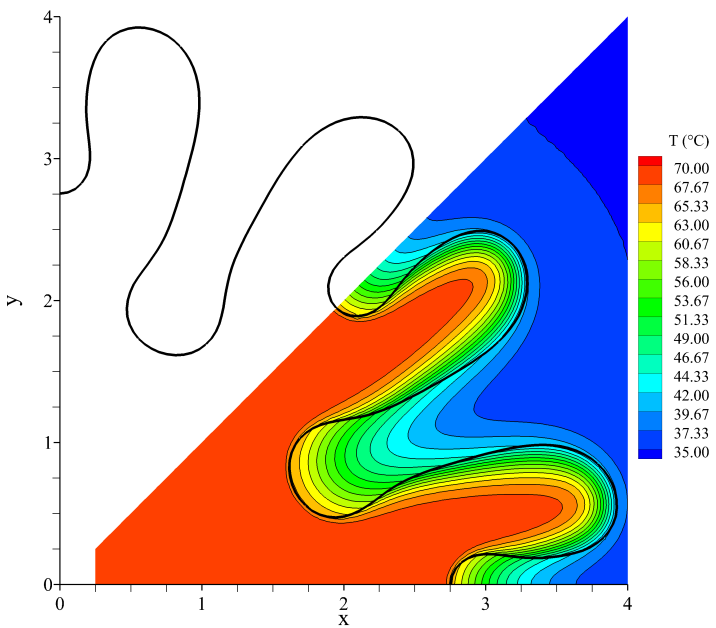

(d) $70^{\circ} \mathrm{C}-35^{\circ} \mathrm{C}$

Figure 7: (a) and (b) Temperature scatter plots around the interface for the hot and cold injection cases at $t=30$. (c) and (d) Temperature contour plots at $t=30$. The bold black interfaces overlay temperature contour plots with thin contour lines of the temperature field. $Q=0.75 \mathrm{~cm}^{2} / \mathrm{s}$.

viscosity for the $\mathrm{CO}_{2}$ and brine. In contrast, the local mobility ratio is decreased for the hot injection case as the temperature drops at the interface. In isothermal radial viscous fingering, as the mobility ratio is increased for a fixed global capillary number, the base should show more stagnation and perturb less into the domain. However, here the opposite effect is seen, where a more displaced finger base is observed in the cold injection case in comparison to the hot injection, with an associated increase in the local mobility ratio. Also, the hot injection cases all show near identical base movement to each other, even though the local mobility ratio (and capillary number) is changing. This indicates that the base movement in non-isothermal regimes cannot be sufficiently described by only considering the local mobility ratio and capillary number, as is the case in isothermal displacements.

The increased movement of the cold injection case in comparison to the hot injection case is due to the difference in homogeneous perturbed pressures. At the finger bases, the perturbed pressure gradient contributes only a small amount, with the primary movement being controlled by the homogeneous pressure gradient. At lower injection temperatures, the finger base homogeneous pressure gradient is larger (see Figure $5(\mathrm{~d})$ ), increasing the growth rate and displacement in comparison to hot injection cases. 


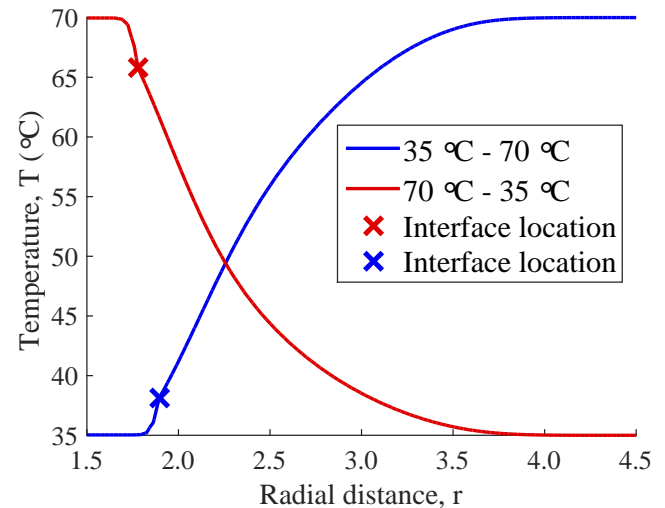

(a) Finger base temperature

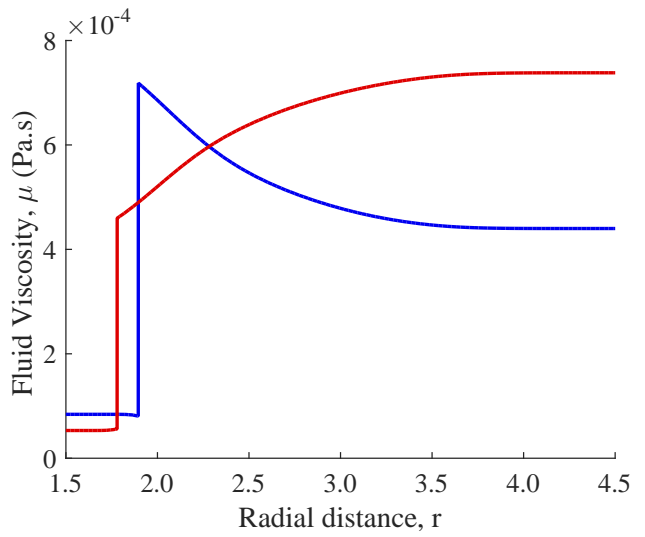

(c) Finger base viscosity

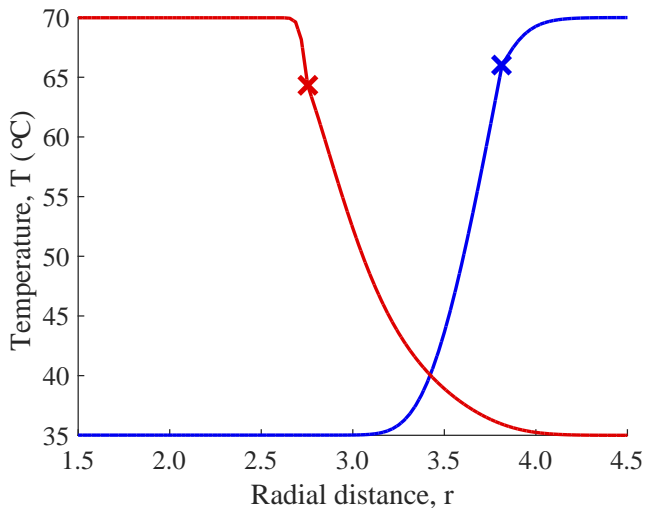

(b) Finger front temperature

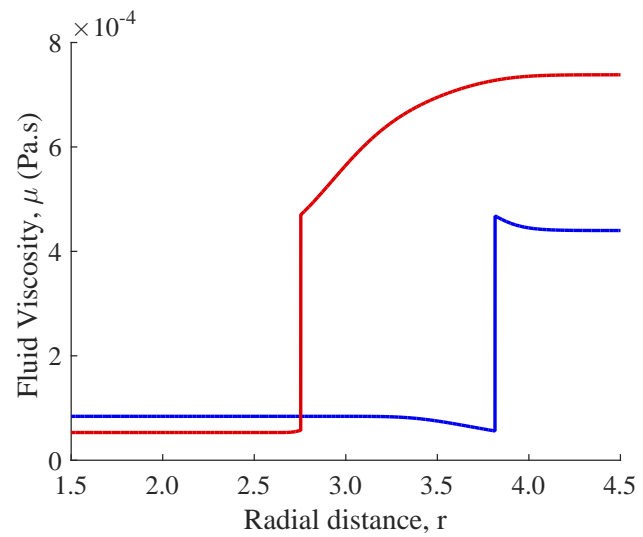

(d) Finger front viscosity

Figure 8: Temperature and viscosity profiles taken parallel to the finger front (along $y=0$ ) and base (along $y=x \cdot \tan (\pi / 8)$ at $t=30, Q=0.75 \mathrm{~cm}^{2} / \mathrm{s}$. Legend for all plots shown in (a).

In cases with equal injection temperatures (Figure 4(a) and (c)), the homogeneous pressure gradients are also equal. This means an increased base mobility in the $35^{\circ} \mathrm{C}-50^{\circ} \mathrm{C}$ and $35^{\circ} \mathrm{C}-70^{\circ} \mathrm{C}$ cases raises the growth rate and displaces the base further into the domain.

If the injection temperature is increased (Figure 4(b) and (d)), the homogeneous pressure gradient decreases. When this is coupled with the corresponding increase in mobility due to the higher temperature, very similar growth rates and base displacements are exhibited. The base movement in non-isothermal cases is therefore controlled mainly by the resident temperature; for equal resident temperatures the base movement is identical and when the resident temperature is increased there is a corresponding increase in the growth rate and base displacement.

\subsection{Thermo-viscous fingering with a continuous viscosity profile}

To further explore the effect of hot/cold injection on the thermo-viscous fingering regime we present immiscible displacement results using injection and resident fluids with the same base viscosities, $\bar{\mu}_{1}=\bar{\mu}_{2}$ and the same dependence on the temperature field $\alpha_{1}=\alpha_{2}$. This means that the viscosity profile is continuous across the immiscible interface, with smooth variations coming purely from the transient temperature field. There still exists surface tension between the fluids, but the effects of the thermal viscosity profile can be analysed independently from a discontinuous viscosity profile generated by the fluids.

In Figure 9, the interfacial displacement for the continuous viscosity profile regime can be seen for the different temperature cases. Here, we use the base viscosity and $\alpha$ values of brine at the corresponding injection temperatures for both injection and resident fluids. The interfacial evolutions shows that the cold injection case quickly stabilises the initial perturbation and displaces as a circle. Without a discontinuous viscosity driving the growth of perturbations, the interface is 


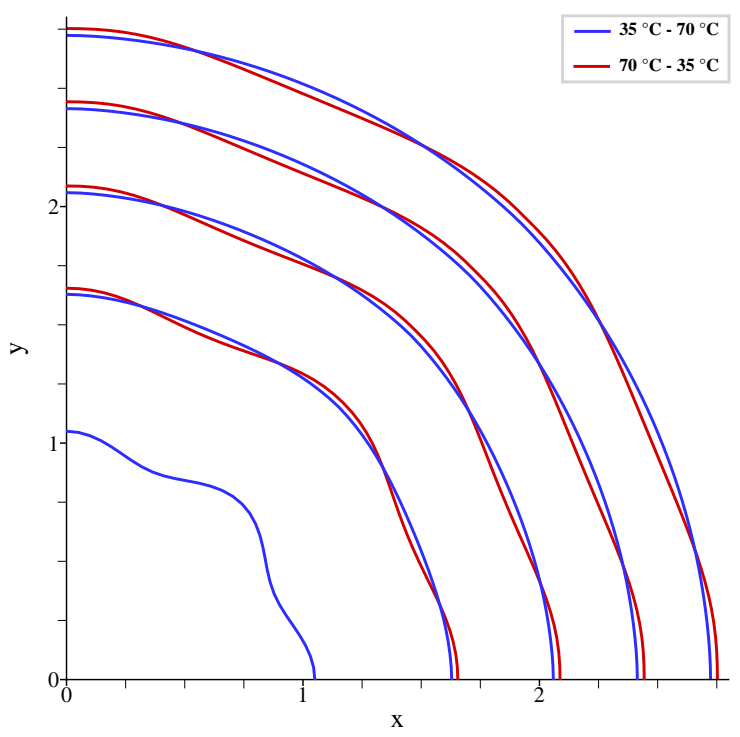

Figure 9: Interface plots at increments of $\Delta t=5$ from $0 \leq t \leq 20$ for non-isothermal injection regimes using fluids with the same base viscosities and dependence on temperature. $Q=0.75 \mathrm{~cm}^{2} / \mathrm{s}$.

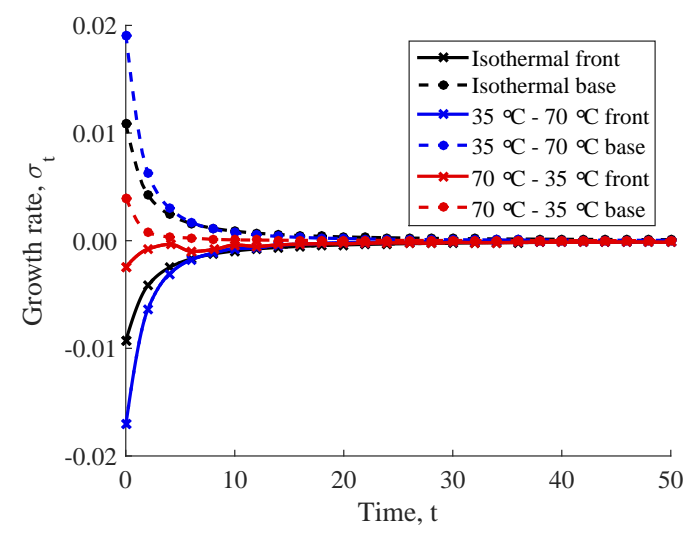

(a)

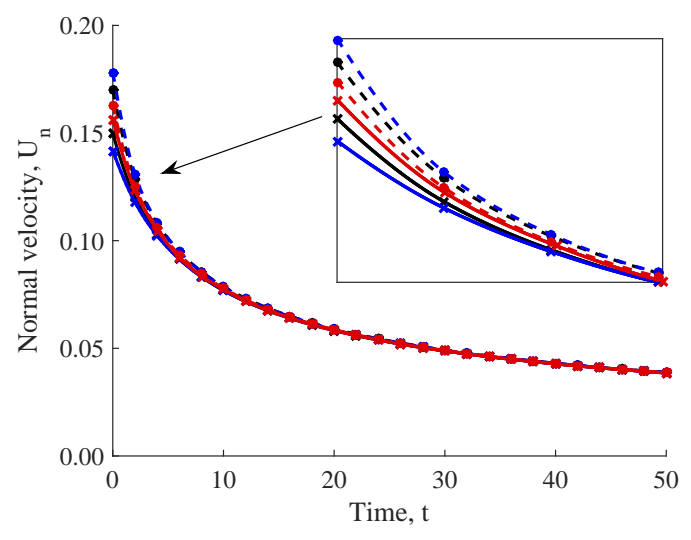

(b)

Figure 10: Plots showing (a) Finger base and front growth rate with time. (b) Finger base and front normal velocity with time. Legend for both plots shown in (a).

stabilised by the surface tension. However, in the hot injection case the perturbation is not damped as significantly and appears to remain with a constant amplitude after $t=15$. The perturbation amplitude changes initially on the first plots when $t \leq 10$ but then seems to stabilised to a constant value afterwards.

The growth rate and velocity plots in Figure 10 show the short and long term behaviour of the different temperature regimes. Initially, the front of the cold injection case in Figure 10(a) experiences a smaller growth rate than the isothermal case, with the base experiencing a larger growth rate. This causes the interface to quickly stabilise whilst displacing to form a circle. However, the hot injection case exhibits a higher growth rate at the front and a lower growth rate at the base compared to the isothermal case, indicating that it maintains some of its initial perturbation and works against the surface tension to maintain the perturbed shape. With time, the growth rate of the fronts and bases for all cases drops to zero, and they evolve with equal velocity at the front and base, shown in Figure 10(b). This means that the interfaces are simply convected with the shape they formed in the initial growth when $0 \leq t \leq 10$.

When the growth rate drops to zero in the hot injection case, there is still a contribution to the growth rate from the perturbed velocity; enough to maintain the perturbation amplitude. The 
source term velocity is larger at the finger base compared to the finger tip (since it is closer to the injection source), meaning the perturbed velocity component must be smaller at the base so that the total velocity is equal along the interface. If the perturbed velocity component was equal along the interface, or its sign reversed (as in the cold injection case), the interface would simply form a circle.

The non-isothermal temperature regimes when considered independently without a discontinuous viscosity profile are neutrally stable. The hot injection case is able to reduce the stabilisation effects from the surface tension around the interface, with some of the initial perturbation being maintained. However, the perturbation does not grow with time even at this high injection temperature, and the growth rate of the perturbation eventually falls to zero.

Thermal effects that vary the viscosity profile in a smooth manner act to promote or hinder the existing viscous fingering regime, but alone do not promote the onset of viscous fingering. This is exemplified in the previous section, where thermal effects enhanced the viscous fingering bifurcation in the hot cases and hindered the fingering in the cold cases. The stabilisation from the cold cases was never enough to completely hinder the finger growth, and once the temperature field had sufficiently diffused the growth rate of features with similar curvatures tended to towards the same value.

\subsection{Thermal diffusivity effects}

In this final section, we explore the effect that the thermal diffusivity has on the thermo-viscous fingering problem. Up until this point, we have used the physical diffusivities of $\mathrm{CO}_{2}$ and brine for all simulations. The diffusivity controls the sharpness of the mobility profile (affecting the acceleration/deceleration from the perturbed pressure) and the distribution of mobility around the interface controlling the bifurcation mode, meaning it is key to the entire thermo-viscous fingering process. Here, we present four diffusivity cases using the $50^{\circ} \mathrm{C}-35^{\circ} \mathrm{C}$ and $35^{\circ} \mathrm{C}-50^{\circ} \mathrm{C}$ injection regime with $Q=0.75$, summarised in Table $4 . \beta_{d}=D_{1} / D_{2}$ is the ratio of the diffusivities, and $P e$ is the Peclet number using the diffusivity of the inner zone.

We keep the fluid properties equal between diffusivity cases for a given temperature regime, only varying the diffusivity. Diffusivity case 1 represents the base case with the physical $\mathrm{CO}_{2}$ and brine diffusivities. The diffusivities in each zone are swapped in diffusivity case 2, and in case 3 they are made equal representing a single-zone heat transfer problem. In case 4 , we increase the diffusivity in each zone by a factor of 10 compared to case 1 to showcase a highly diffuse temperature field regime and its impact on the fingering morphology.

\begin{tabular}{|c|c|c|c|c|}
\hline $\begin{array}{c}\text { Diffusivity } \\
\text { case }(\mathrm{DC})\end{array}$ & $D_{1}\left(\mathrm{~cm}^{2} / \mathrm{s}\right)$ & $D_{1}\left(\mathrm{~cm}^{2} / \mathrm{s}\right)$ & $\beta_{d}$ & $P e$ \\
\hline 1 & 0.0005 & 0.0015 & 0.333 & 1500 \\
\hline 2 & 0.0015 & 0.0005 & 3.000 & 500 \\
\hline 3 & 0.0015 & 0.0015 & 1.000 & 500 \\
\hline 4 & 0.0050 & 0.0150 & 0.333 & 150 \\
\hline
\end{tabular}

Table 4: Thermal diffusivity cases with associated fluid properties.

The interfacial plots at $t=30$ for different diffusivity cases can be seen in Figure 11 . In both temperature regimes, raising the inner diffusivity in cases 2,3 and 4 has led to a change in the bifurcation mode compared to case 1. During cold injection, the bifurcation mode transitions to a two finger split, whereas in the hot injection case it transitions to a three finger split. As the resident temperature remains equal throughout all cases, the base positions have remained entirely unaffected by changing the diffusivity regime.

When the thermal diffusivity is raised in both zones by a factor of 10 (compared to the base case), the growth rates tend to that of the isothermal case, shown in Figure 12(a) and (b). In the early time frame, we see a very similar growth rate (almost identical) for diffusivity cases 1,2 and 3 where the diffusivities are in the same order of magnitude.

Diffusivity case 4 shows a very similar initial growth (and long term growth in the hot injection case) to the isothermal case, as the temperature (and hence mobility) field is highly diffuse causing only minor acceleration/deceleration effects with the induced perturbed pressure gradient. This is 


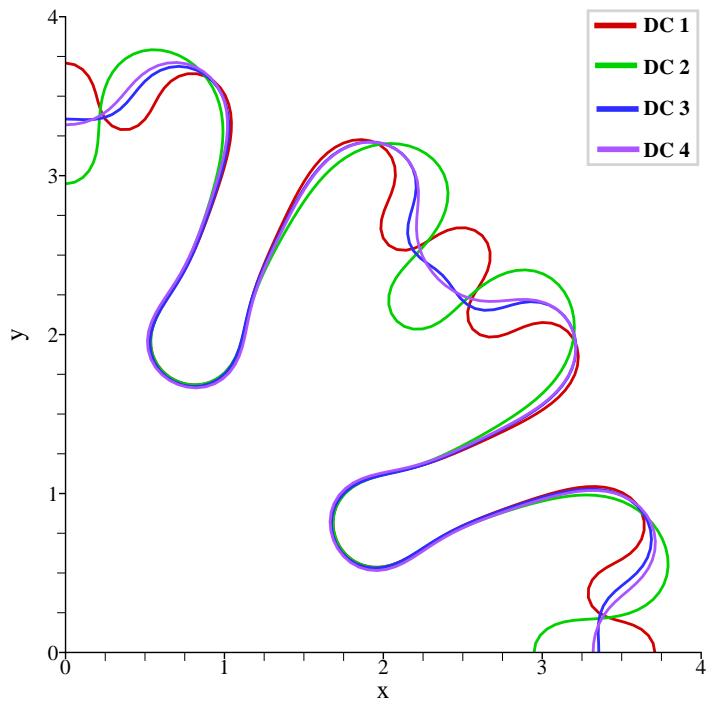

(a) $35^{\circ} \mathrm{C}-50^{\circ} \mathrm{C}$

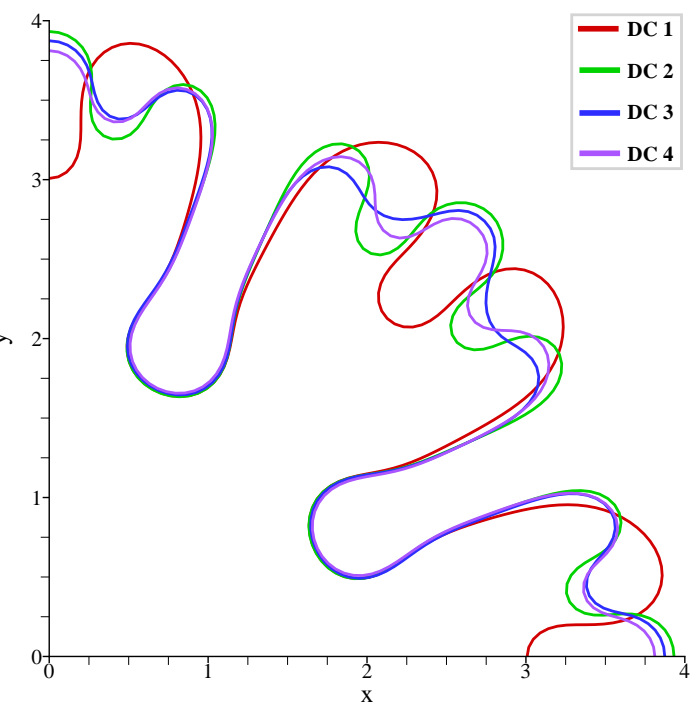

(b) $50^{\circ} \mathrm{C}-35^{\circ} \mathrm{C}$

Figure 11: Interface plots at $t=30$ for hot and cold injection regimes with different diffusivity parameters.

as expected, since the non-isothermal should tend towards the isothermal with highly diffuse temperature fields. Although the growth rate is initially similar to the isothermal in the cold injection case, after time the growth rate of the cold case drops, as the distribution of temperature in the domain causes the sides of the front to be accelerated more strongly than the tip. The temperature contours at the late stage show very little resemblance to the interfacial pattern, spreading radially from the injection source (see Figure 13(c)). Since the interface and the associated convective velocity field has little effect on the transport of the temperature field, the interface displaces with a radially diffusing temperature field, meaning the finger sides evolve in regions of higher mobility regions than the tip. This enhances the spreading mechanism and causing a two finger bifurcation. The reverse can be seen in Figure 13(d), where the finger sides in the hot injection cases evolve into low mobility regions, promoting the growth of the finger tip and the three finger bifurcation.

The same mechanism also controls the bifurcation mode in diffusivity case 2 and 3 , in which the increased diffusivity in the inner zone promotes heat transfer. Figure 13(a) and (b) show the diffuse inner regions for diffusivity case 2 at the two temperature regimes (note that very similar plots are obtained for diffusivity case 3, with a greater spacing between contour lines in zone 2). In the hot injection case, the temperature contours of the diffuse inner region follow the interface less closely in comparison to case 1, meaning that regions of the interface closer to the injection source displace with a higher temperature and mobility. This causes the tip to be be accelerated and the interface bifurcates into three fingers. In contrast, the cold injection case with reversed diffusivites has a colder region near the injection source that propagates radially outwards (when the diffusivity is raised). This means the finger tips are closer to the low temperature source when the finger initially spreads and are decelerated in comparison to the finger sides, forming the two finger bifurcation. We see a switch in the bifurcation mode in the high inner diffusivity cases here as temperature field less closely resembles the interface, instead propagating radially outwards from the source temperature, following a more circular evolution that produces non-uniformity around the interface.

Two effects are caused by the changing diffusivity, firstly, by raising the inner diffusivity (and lowering the Peclet number) the bifurcation mode is altered by forcing the temperature field to evolve in a more circular, radial fashion less closely resembling the interfacial pattern. This creates non-uniformities in the mobility field that don't match the interfacial advection, causing the bifurcation mode to switch from that given by the high Peclet number cases. Secondly, by changing the diffusivity ratio, the interfacial temperature can be raised/lowered without changing the bifurcation mode, instead changing the growth rate. In hot injection cases, by decreasing $\beta_{d}$, heat transfer is promoted in the outer zone and the temperature at the interface is lowered, hindering 


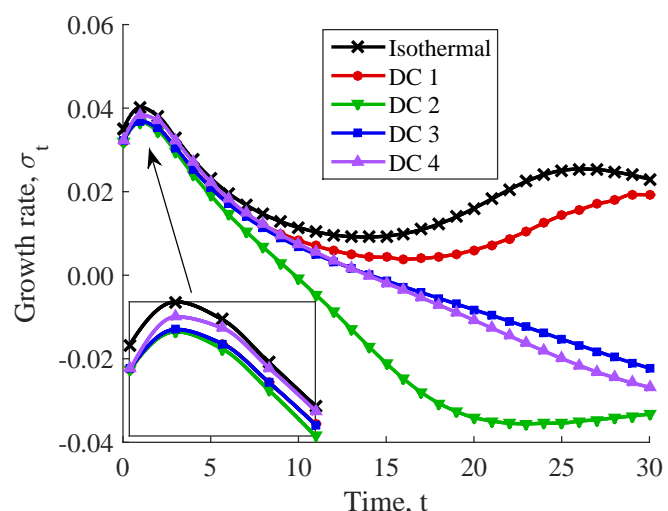

(a) $35^{\circ} \mathrm{C}-50^{\circ} \mathrm{C}$

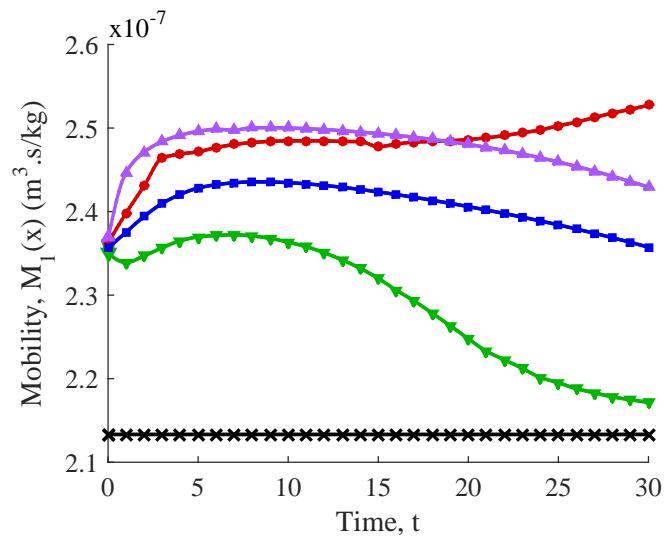

(c) $35^{\circ} \mathrm{C}-50^{\circ} \mathrm{C}$

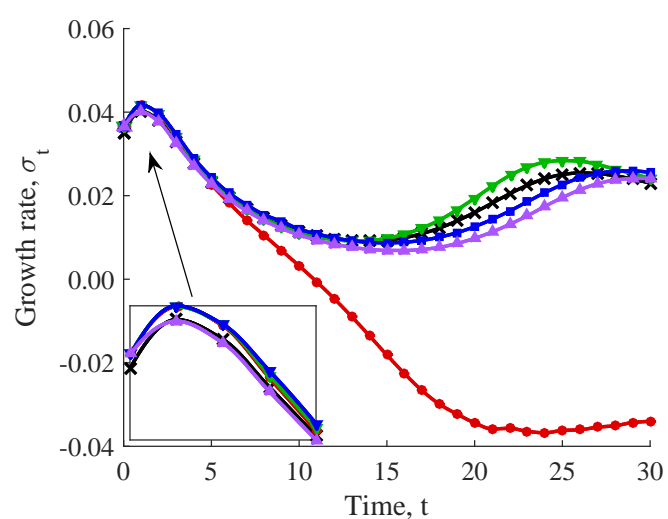

(b) $50^{\circ} \mathrm{C}-35^{\circ} \mathrm{C}$

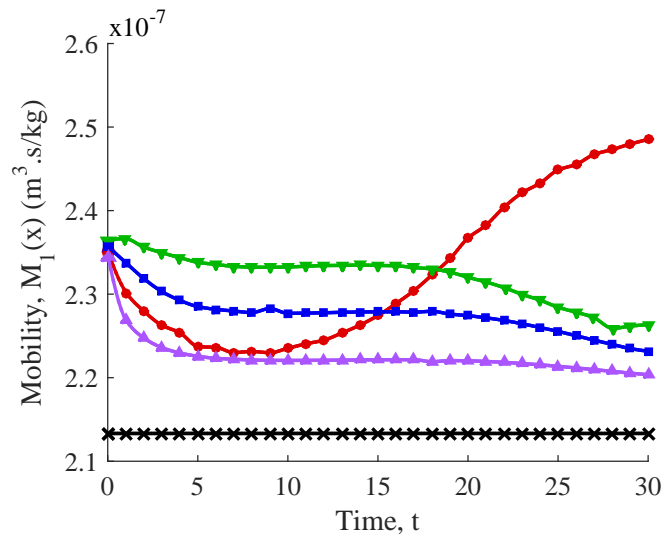

(d) $50^{\circ} \mathrm{C}-35^{\circ} \mathrm{C}$

Figure 12: Timeseries plots of the growth rate and mobility at the finger fronts for the hot and cold injection regimes with different diffusivity parameters. $Q=0.75$.

the three finger interfacial growth. In cold cases, decreasing $\beta_{d}$ also enhances heat transfer in the outer region, but when coupled with the reversed temperature gradient, this increases the front temperature and hinders the two finger growth.

By lowering the thermal Peclet number, we see similar effects to those presented in [18] and [20] for miscible regimes; the stabilising/destabilising effects of a cold/hot injection regime are reduced. [18] and [20] also generate similar results with the inclusion of thermal lag, which acts to slow the convective velocity in the heat transfer compared to that in the solutal regime. As well as changing the location of the thermal front, this also effective reduces the thermal Peclet number, with very similar qualitative results to those presented here. We mention these miscible results to provide some comparison to previous works in the area of thermo-viscous fingering, but note that although qualitatively similar, the mechanism for their formation are entirely different. 


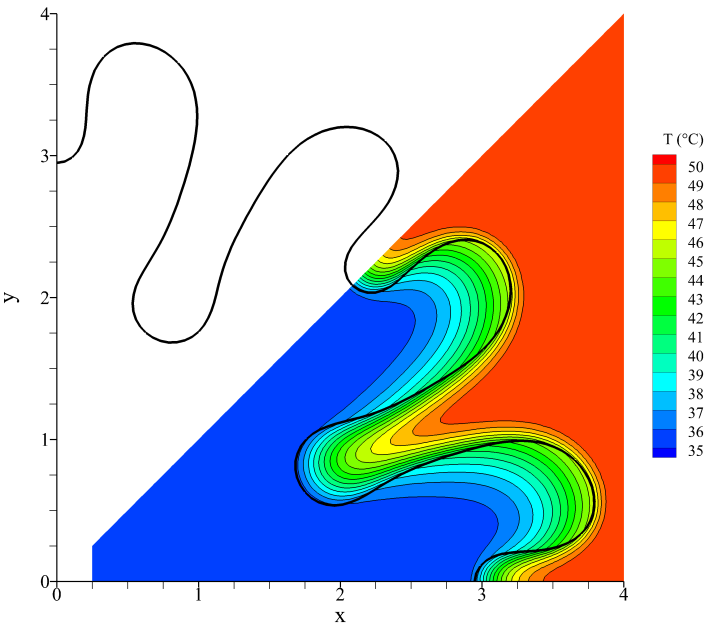

(a) DC $2,35^{\circ} \mathrm{C}-50^{\circ} \mathrm{C}$

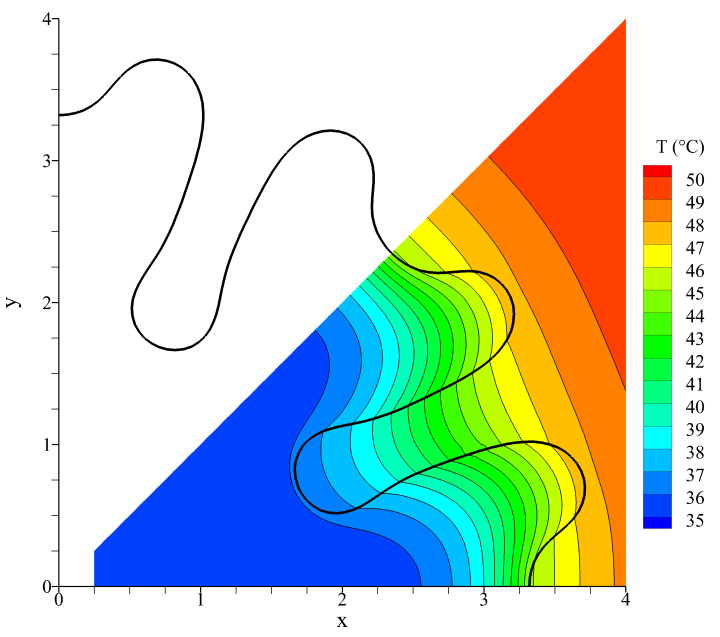

(c) $\mathrm{DC} 4,35^{\circ} \mathrm{C}-50^{\circ} \mathrm{C}$

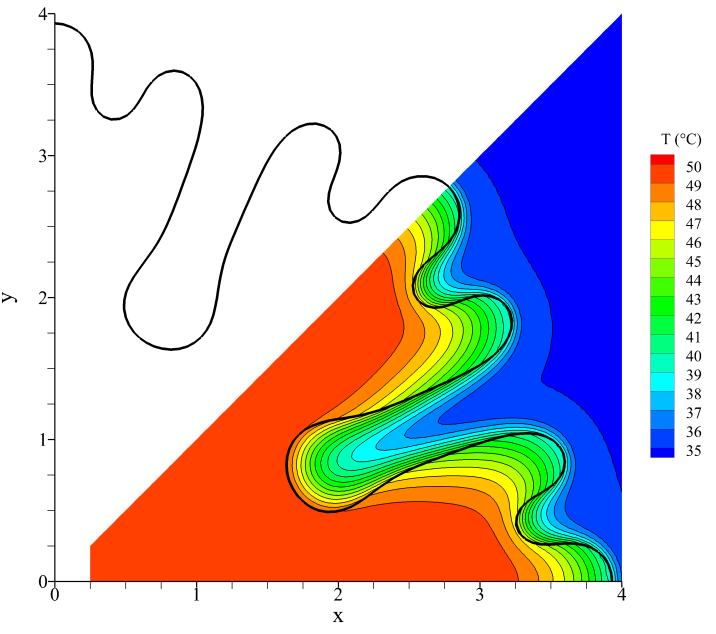

(b) $\mathrm{DC} 2,50^{\circ} \mathrm{C}-35^{\circ} \mathrm{C}$

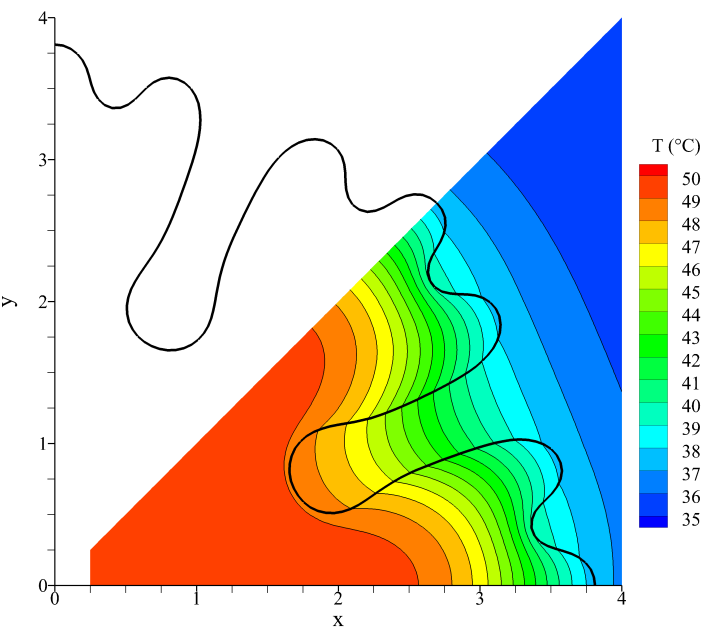

(d) $\mathrm{DC} 4,50^{\circ} \mathrm{C}-35^{\circ} \mathrm{C}$

Figure 13: Temperature contour plots for diffusivity case 2 (top) and 4 (bottom) at $t=30$. The thick black lines show the interface, with thin black lines representing contour lines. $Q=0.75$.

\section{Conclusion}

In this work, the thermo-viscous fingering problem was investigated during the displacement of immiscible fluids using a high resolution BEM-RBF-FC method. The numerical method utilised mesh reduction techniques to solve the coupled heat transfer and Darcy flow problem in an accurate and efficient manner. These new methods allowed the solution to moving multi-zone problems without extrapolation, utilising the truly meshless nature of the RBF-FC method to its full extent. The physical mechanisms that control the immiscible thermo-viscous fingering regime were investigated in a systemic manner, revealing the follow system behaviour:

- In high Peclet number flows, the early stage growth rate is governed by the pressure gradient at the interface. For hot injection cases, the magnitude of the pressure gradient is increased, due to an additive perturbed pressure gradient from the decreasing mobility field. When combined with an increased mobility, this enhances the growth rate. In cold injection cases, the growth rate is hindered due to a subtractive perturbed pressure gradient resulting from an increasing mobility field.

- The late stage bifurcation regime is controlled primarily by the mobility distribution around the interface. When the mobility decreases towards the finger tip, a two finger bifurcation 
is promoted, whereas when the mobility increases towards the tip a three finger bifurcation can be achieved if the injection flux is high enough.

- The diffusivity and diffusivity ratio control the mobility distribution around the interface. When the inner diffusivity is increased, the temperature field more closely resembles radial diffusion from a point source, which promotes two finger bifurcation in cold injection regimes and three finger bifurcations in hot regimes. By increasing the diffusivity ratio, the temperature and mobility are increased at the interface for hot injections, and decreased for cold injections.

- Finger base displacement is controlled by the resident fluid temperature. When the resident temperature is increased for a given flow rate, the finger base growth increases. For a fixed resident temperature with varying injection temperature, the base growth rates are equal.

- Thermal effects in the fingering evolution are neutrally stable. They act to enhance or reduce existing perturbations that grow due to the fluids' homogeneous viscosity contrast. However, thermal effects are not enough alone to grow perturbations and cause a fingering instability without an existing viscosity contrast. Thermal effects diminish with time as the temperature field diffuses, with classical viscous fingering dynamics eventually overcoming any stabilisation from a smoothly decreasing mobility field.

With these findings, careful variation of the fluid parameters and initial conditions could control the thermo-viscous fingering instability in a predictable manner, creating alternative bifurcation modes that cannot be predicted using classical viscous fingering theory at corresponding isothermal temperatures. This study represents the first of its kind in identifying the key mechanisms behind immiscible thermo-viscous fingering and hopes to provide a baseline for future research.

\section{Acknowledgements}

The authors would like to thank the University of Nottingham HPC team for the use of the Minerva supercomputer cluster. The present work has been supported by an EPSRC post-graduate research scholarship.

\section{References}

[1] A. Shafiei and M.B. Dusseault. Geomechanics of thermal viscous oil production in sandstones. Journal of Petroleum Science and Engineering, 103:121 - 139, 2013.

[2] S.J. Zarrouk A. McDowell and R. Clarke. Modelling viscous fingering during reinjection in geothermal reserviors. In Proceedings World Geothermal Congress 2015, Melbourne, Australia, 2015.

[3] Y. Wang, C. Zhang, N. Wei, M. Oostrom, T.W. Wietsma, X. Li, and A. Bonneville. Experimental study of crossover from capillary to viscous fingering for supercritical $\mathrm{CO}_{2}$ - water displacement in a homogeneous pore network. Environmental Science and Technology, 47:212 $-218,2013$.

[4] G.M. Homsy. Viscous fingering in porous media. Annual Review of Fluid Mechanics, 19:271$311,1987$.

[5] V. Vilarrasa, S. Olivella, J. Carrera, and J. Rutqvist. Long term impacts of cold $\mathrm{CO}_{2}$ injection on the caprock integrity. International Journal of Greenhouse Gas Control, 24:1 - 13, 2014.

[6] R.C Bissell, D.W. Vasco, M. Atbi, M. Hamdani, M. Okwelegbe, and M.H. Goldwater. A full field simulation of the In Salah gas production and $\mathrm{CO}_{2}$ storage project using a coupled geo-mechanical and thermal fluid flow simulator. Energy Procedia, 4:3290 - 3297, 2011.

[7] H. Liu, Z. Hou, P. Were, X. Sun, and Y. Gou. Numerical studies on $\mathrm{CO}_{2}$ injection - brine extraction process in a low - medium temperature reservoir system. Environmental Earth Science, 73:6839 - 6854, 2015. 
[8] F. Moller, A. Liebscher, S. Martens, C. Schmidt-Hattenberger, and M. Streibel. Injection of $\mathrm{CO}_{2}$ at ambient temperature conditions - Pressure and temperature results of the 'cold injection' experiment at the Ketzin pilot site. Energy Procedia, 2014:6298 - 6297, 63.

[9] A. Kopp, A. Bielinski, A. Ebigbo, H. Class, and R. Helmig. Numerical investigation of temperature effects during the injection of carbon dioxide into brine aquifers. In The Eighth International Conference on Greenhouse Gas Control Technologies, Trondheim, Norway., 2006.

[10] M. Sajjadi and J. Azaiez. Thermo-viscous fingering in heterogeneous media. In SPE Heavy Oil Conference Canada, 12-14 June, Calgary, Alberta, Canada, 2012.

[11] S.H. Davis. Thermocapillary instabilities. Annual review in Fluid Mechanics, 19:403 - 435, 1987.

[12] W. Boos and A. Thess. Thermocapillary flow in a Hele-Shaw cell. Journal of Fluid Mechanics, 352:305 - 330, 1997.

[13] D Stevens, A LaRocca, H Power, and V LaRocca. A Generalised RBF Finite Difference Approach to Solve Nonlinear Heat Conduction Problems, on Unstructured Datasets, chapter 13, pages 281-296. InTech, 2011.

[14] D. Pritchard. The instability of thermal and fluid fronts during radial injection in a porous medium. Journal of Fluid Mechanics, 508:133 - 163, 2004.

[15] D. Pritchard. The linear stability of double - diffusive miscible rectilinear displacements in a Hele-Shaw cell. Eureopean Journal of Mechanics B/Fluids, 28:564 - 577, 2009.

[16] M. Mishra, P.M.J Trevelyan, C. Almarcha, and A. De Wit. Influence of double diffusive effects on miscible viscous fingering. Physical Review Letters, 105:204501, 2010.

[17] T. Sheorey and K. Muralidhar. Isothermal and non-isothermal oil-water flow and viscous fingering in a porous medium. International Journal of Thermal Sciences, 42:665 - 676, 2003.

[18] M.N. Islam and J. Azaiez. Miscible thermo-viscous fingering instability in porous media. Part 2: Numerical simulations. Transport in Porous Media, 84:845 - 861, 2010.

[19] M.N. Islam and J. Azaiez. Thermo-viscous fingering in quarter five-spot miscible displacements. European Journal of Mechanics B/Fluids, 30:107 - 119, 2011.

[20] M. Sajjadi and J. Azaiez. Dynamics of fluid flow and heat transfer in homogeneous porous media. The Canadian Journal of Chemical Engineering, 91:687 - 697, 2013.

[21] K.E. Holloway and J.R. de Bruyn. Numerical simulations of a viscous-fingering instability in a fluid with a temperature-dependent viscosity. Canadian Journal of Physics, 84:273 - 287, 2006.

[22] Y. Nagatsu, N. Fujita, Y. Kato, and Y. Tada. An experimental study of non-isothermal miscible displacements in a Hele-Shaw cell. Experimental Thermal and Fluid Science, 33:695 $-705,2009$.

[23] K.E. Holloway and J.R. de Bruyn. Viscous fingering with a single fluid. Canadian Journal of Physics, 83:551 - 564, 2005.

[24] C.W. Park and G.M. Homsy. Two phase displacement in Hele-Shaw cells: Theory. Journal of Fluid Mechanics, 139:291 - 308, 1984.

[25] E. Alvarez-Lacelle, J. Ortin, and J. Casademunt. Relevance of dynamic wetting in viscous fingering patterns. Physical Review E, 74:025302(R), 2006.

[26] P.H. Anjos and J.A. Miranda. Radial viscous fingering: Wetting effects on pattern-forming mechanisms. Physical Review E, 88:053003, 2013. 
[27] S.J. Jackson, D. Stevens, D. Giddings, and H. Power. Dynamic-wetting effects in finitemobility-ratio Hele-Shaw flow. Physical Review E, 92:023021, 2015.

[28] M.N. Islam and J. Azaiez. Miscible thermo-viscous fingering instability in porous media. Part 1: Linear stability analysis. Transport in Porous Media, 84:821 - 844, 2010.

[29] S.J. Jackson, H. Power, D. Giddings, and D. Stevens. The stability of immiscible viscous fingering in Hele-Shaw cells with spatially varying permeability. Computer Methods in Applied Mechanics and Engineering, In press, March 2017.

[30] S.J. Jackson, D. R. Stevens, H. Power, and D. Giddings. A boundary element method for the solution of finite mobility ratio immiscible displacement in a Hele-Shaw cell. International Journal for Numerical Methods in Fluids, 78(9):521 - 551, 2015.

[31] D. Stevens, H. Power, C.Y. Meng, and D. Howard. An alternative local collocation strategy for high-convergence meshless PDE solutions, using radial basis functions. Journal of Computational Physics, 245:52 - 75, 2013.

[32] S.J. Jackson, D. Stevens, D. Giddings, and H. Power. An adaptive RBF finite collocation approach to track transport processes across moving fronts. Computers and Mathematics with Applications, 71(1):278 - 300, 2016.

[33] D. Stevens, H. Power, M. Lees, and H. Morvan. A local Hermitian RBF meshless numerical method for the solution of multi-zone problems. Numerical Methods for Partial Differential Equations, 27(5):1201 - 1230, 2011.

[34] T.D. Aslam. A partial differential equation approach to multidimensional extrapolation. Journal of Computational Physics, 193:349 - 355, 2003.

[35] A. Fenghour, W.A. Wakeham, and V. Vesovic. The viscosity of carbon dioxide. Journal of Physical and Chemical Reference Data, 27(1):31 - 44, 1998.

[36] J. Kestin, H.E. Khalifa, and R.J. Correia. Tables of the dynamic and kinematic viscosity of aqueos $\mathrm{NaCL}$ solutions in the temperature range $20-150^{\circ} \mathrm{C}$ and the pressure range $0.1-35$ MPa. Journal of Physical and Chemical Reference Data, 10:71 - 87, 1981.

[37] S. Bachu and D.B. Bennion. Interfacial Tension between $\mathrm{CO}_{2}$, Freshwater, and Brine in the Range of Pressure from 2 to $27 \mathrm{MPa}$, Temperature from 20 to $125^{\circ} \mathrm{C}$, and Water Salinity from 0 to $334000 \mathrm{mg} / \mathrm{L}$. Journal of Chemical Engineering Data, 54:765-775, 2009.

[38] E.W. Lemmon, M.O. McLinden, and D.G. Friend. NIST Chemistry WebBook, NIST Standard Reference Database Number 69, chapter Thermophysical Properties of Fluid Systems. National Institute of Standards and Technology, Gaithersburg MD.

[39] M Van Genuchten and W Alves. Analytical solutions of the one-dimensional convectivedispersive solute transport equation. Technical report, United States Department of Agriculture, Agricultural research service, Technical bulletin number 1661, 1982.

[40] D.K. Jaiswal, A. Kumar, and R.R. Yadav. Analytical solution to the one - dimensional advection - diffusion equation with temporally dependent coefficients. Journal of Water Resource and Protection, 3:76 - 84, 2011.

[41] O.K Jensen and B.A. Finlayson. Solution of transport equations using a moving coordinate system. Advances in Water Resources, 3:9 - 18, 1980.

[42] Y. Sun and I.S. Wichman. On transient heat conduction in a one-dimensional composite slab. International Journal of Heat and Mass Transfer, 47:1555 - 1559, 2004.

[43] F. de Monte. Transient heat conduction in a one-dimensional composite slab. A natural analytic approach. International Journal of Heat and Mass Transfer, 43:3607 - 3619, 2000. 


\section{Appendix}

\section{A1. The auxiliary multi-zone RBF-FC method}

The multi-zone convection-diffusion problems under consideration in this work (governed by equation (12) and (22)) can be described generally as initial boundary value problems:

$$
\begin{aligned}
& \frac{\partial \phi_{l}(\boldsymbol{x}, t)}{\partial t}=L_{l}\left[\phi_{l}(\boldsymbol{x}, t)\right]+S_{l}(\boldsymbol{x}, t) \quad \boldsymbol{x} \in \Omega_{l} \\
& \phi_{l}(\boldsymbol{x}, 0)=p_{l}(\boldsymbol{x}) \quad \boldsymbol{x} \in \Omega_{l} \\
& B_{l}\left[\phi_{l}(\boldsymbol{x}, t)\right]=g^{l}(\boldsymbol{x}) \quad \boldsymbol{x} \in \partial \Omega_{l} \\
& C_{1}\left[\phi_{1}(\boldsymbol{x}, t)\right]-C_{2}\left[\phi_{2}(\boldsymbol{x}, t)\right]=h(\boldsymbol{x}) \quad \boldsymbol{x} \in \partial \Omega_{\text {int }} \\
& Q_{1}\left[\phi_{1}(\boldsymbol{x}, t]-Q_{2}\left[\phi_{2}(\boldsymbol{x}, t]=f(\boldsymbol{x}) \quad \boldsymbol{x} \in \partial \Omega_{\text {int }}\right.\right.
\end{aligned}
$$

Here, $\Omega_{l}$ is the interior of zone $l, \partial \Omega_{l}$ is the boundary of zone $l$ and $\partial \Omega_{i n t}$ is the fluid-fluid interface. Equation (32) is the initial conditions for the problem. Equations (34) and (35) are the solution and flux matching conditions at the interface. For continuous solution and flux $h(\boldsymbol{x})=f(\boldsymbol{x})=0$. We take a Crank-Nicholson approximation to the time derivative:

$$
\begin{aligned}
\frac{\phi_{l}^{n+1}-\phi_{l}^{n}}{\Delta t}= & \theta L\left[\phi_{l}^{n+1}\right]+(1-\theta) L\left[\phi_{l}^{n}\right] \\
& +S(x, t)
\end{aligned}
$$

To obtain modified PDE governing operators

$$
\begin{aligned}
& \bar{L}=I-\theta \Delta t L \\
& \hat{L}=I+(1-\theta) \Delta t L
\end{aligned}
$$

such that

$$
\bar{L}_{l}\left[\phi_{l}^{n+1}\right]=\hat{L}_{l}\left[\phi_{l}^{n}\right]+S_{l}(x, t)=\bar{S}_{l}(x, t)
$$

In the equations above, $L(\hat{L}$ and $\bar{L})$ and $B$ are linear partial differential operators on the domain $\Omega$ and on the boundary $\partial \Omega$ respectively. By using this type of time stepping algorithm, the transient problem is reduced to a series of inhomogeneous steady-state problems, with the inhomogeneous term a function of the solution at the previous time step. Steady-state problems are achieved by setting $\hat{L}$ to zero and $\bar{L}=L$ in the above equations.

The Hermitian collocation approach constructs the value of the solution $\phi$ as a weighted sum of partial differential operators applied to a set of radial basis functions that are centred on nodes $\xi_{j}$. In the Hermitian RBF interpolation in equation (39), the boundary operator $B$ is applied at the domain boundary, the governing PDE $\bar{L}$ operator is applied at the domain interior, and the flux and solution operators $Q$ and $C$ are applied at the multi-zone interface:

$$
\begin{array}{r}
\phi_{l}^{n+1}(\boldsymbol{x})=\sum_{j=1}^{N B} \alpha_{j} B_{l, \xi} \Psi\left(\left\|\boldsymbol{x}-\boldsymbol{\xi}_{j}\right\|\right)+\sum_{j=N B+1}^{N B+N I+1} \alpha_{j} \bar{L}_{l, \xi} \Psi\left(\left\|\boldsymbol{x}-\boldsymbol{\xi}_{j}\right\|\right) \\
+\sum_{j=N B+N I+2}^{N B+N I+N F+2} \alpha_{j} C_{l, \xi} \Psi\left(\left\|\boldsymbol{x}-\boldsymbol{\xi}_{j}\right\|\right)+\sum_{j=N B+N I+N F+3}^{N B+N I+2 N F+3} \alpha_{j} Q_{l, \xi} \Psi\left(\left\|\boldsymbol{x}-\boldsymbol{\xi}_{j}\right\|\right)
\end{array}
$$

In equation (39), $N B$ is the number of boundary nodes, $N F$ is the number of flux (interface) nodes and $N I$ is the number of internal nodes. The RBF operators in equation (39) are Hardy Multiquadric RBF interpolants $\Psi(r)=\left(r^{2}+c^{2}\right)^{\frac{1}{2}}$. These are favored over Gaussian or thin plate spline RBFs due to their accuracy when solving convection-diffusion type problems and the variable shape parameter which can be fine-tuned for specific problems. We use a global, nondimensional version of the shape parameter $c^{*}$, which is related to the local shape parameter $c$ through $c^{*}=c / \Delta$, where $\Delta$ is the average nodal separation in the local stencil. This means the dimensional shape parameter will increase with distance away from the interface as the the nodal separation increases. 


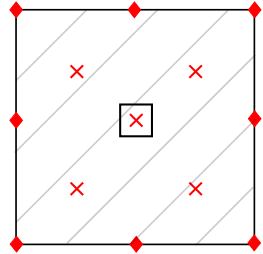

(a)

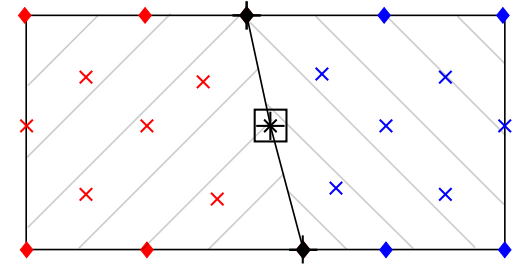

(b)

\begin{tabular}{|cl|}
\hline Zone $1 /$ Zone 2 & \\
$\times \times \times$ & Solution centre \\
$+\times$ & PDE centre \\
$\square$ & Flux centre \\
$\square$ & System centrepoint \\
\hline
\end{tabular}

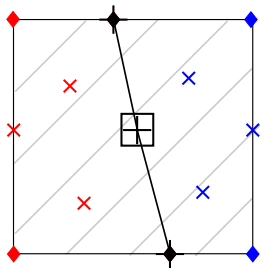

(c)

Figure 14: RBF-FC multi-zone stencil type. (a) Interior stencil. (b) Two embedded multi-zone stencils collocated on the interface. (c) A single auxiliary multi-zone stencil collocated on the interface. Black diagonal lines indicate the fluid-fluid interface. The grey hatching highlights separate RBF stencils.

In the RBF-FC method, the global domain is broken down into a series of overlapping local stencils. These stencils are formed at every interior node in the domain, with three examples shown in Figure 14. By enforcing the PDE system (31 - 35) at a set of test locations, equal to the set of functional centres in a local stencil in zone $l$, a collocation matrix can be formed.

The auxiliary multi-zone method collocates only one stencil at each interface point, enforcing operators from zone 1 and zone 2 in the same matrix system, i.e.

$$
\left[\begin{array}{cccccc}
B_{1, x} B_{1, \xi} & 0 & B_{1, x} \bar{L}_{1, \xi} & 0 & B_{1, x} C_{1, \xi} & B_{1, x} Q_{1, \xi} \\
0 & B_{2, x} B_{2, \xi} & 0 & B_{2, x} \bar{L}_{2, \xi} & B_{2, x} C_{2, \xi} & B_{2, x} Q_{2, \xi} \\
\bar{L}_{1, x} B_{1, \xi} & 0 & \bar{L}_{1, x} \bar{L}_{1, \xi} & 0 & \bar{L}_{1, x} C_{1, \xi} & \bar{L}_{1, x} Q_{1, \xi} \\
0 & \bar{L}_{2, x} B_{2, \xi} & 0 & \bar{L}_{2, x} \bar{L}_{2, \xi} & \bar{L}_{2, x} C_{2, \xi} & \bar{L}_{2, x} Q_{2, \xi} \\
C_{1, x} B_{1, \xi} & -C_{2, x} B_{2, \xi} & C_{1, x} \bar{L}_{1, \xi} & -C_{2, x} \bar{L}_{2, \xi} & C_{1, x} C_{1, \xi}-C_{2, x} C_{2, \xi} & C_{1, x} Q_{1, \xi}-C_{2, x} Q_{2, \xi} \\
Q_{1, x} B_{1, \xi} & -Q_{2, x} B_{2, \xi} & Q_{1, x} \bar{L}_{1, \xi} & -Q_{2, x} \bar{L}_{2, \xi} & Q_{1, x} C_{1, \xi}-Q_{2, x} C_{2, \xi} & Q_{1, x} Q_{1, \xi}-Q_{2, x} Q_{2, \xi}
\end{array}\right] \alpha_{j}=\left[\begin{array}{c}
g_{1, i} \\
g_{2, i} \\
\bar{S}_{1, i} \\
\bar{S}_{2, i} \\
h_{i} \\
f_{i}
\end{array}\right]
$$

In the auxiliary system, the unknown solution fluxes do not appear in the data vector (RHS of (40)), with $f_{i}$ instead enforcing the flux matching condition locally. Similarly, the field variable matching condition appears locally in the form $h_{i}$. The auxiliary system only enforces corresponding zone operators on each other, so that the interpolation is valid in a particular zone. A smooth interpolation cannot be performed using a single interpolating function if there are discontinuities in the operators, hence the need for zone specific interpolations that create a block structure in (40). The PDE centres present at the system centrepoints of the two embedded systems are not apparent in the auxiliary system. This is because the local enforcement of the matching conditions provides a strong collocation at the centrepoint, which already takes into account the multi-zone transport scalars that appear in the PDE centres, so there is no need for a third/fourth collocation here.

For interior stencils, the last two rows/columns of (40) can be dropped, leaving an interpolation that depends only on boundary and PDE operators. By forming an RBF collocation over each of the $N$ stencils, which are formed around each strictly-interior domain node, a series of $N$ local RBF collocation systems are formed given by (40). This is equivalent to defining at each interpolation stencil an auxiliary boundary value problem rather than performing a pure interpolation as is done in the RBF-FD approach.

To link the systems together, $\phi_{l}(\boldsymbol{x})$ is reconstructed at each system centrepoint, thereby obtaining an estimate for the field variable in terms of the corresponding unknown values at the solution centres on the periphery of the stencil. Using the RBF reconstruction formula (39) the approximate value of the field variable $\phi_{l}(\boldsymbol{x})$ may be computed for any system centrepoint $\boldsymbol{x}_{c}^{(k)}$ in the domain. By performing the reconstruction of $\phi_{l}(\boldsymbol{x})$ at the centrepoint of each local system $k$, 
a series of $N$ simultaneous equations are formed for the $N$ unknown values. Since each reconstruction depends only on nodes in the corresponding local system, the resulting global matrix system is sparsely populated.

For the auxiliary method, since the unknown flux terms do not appear in the data vectors, only the values of $\phi_{l}(\boldsymbol{x})$ are obtained from the solution of the sparse global system. The newly computed nodal values of $\phi$ may be fed back into the the local data vectors, allowing the value of $\hat{L}\left[\phi^{n}\right]$ to be reconstructed at the end of each time step. At the first time step the value of $\hat{L}$ is unknown, and must be approximated from the initial solution field. The most straightforward way to obtain this estimate is to perform an initialisation time step of zero size; i.e. setting $\Delta t=0$ and utilising the existing local systems and reconstruction algorithms to perform a simple RBF interpolation of the initial data field in order to obtain $\bar{S}(x, t)$.

\section{A2. Multi-zone verification}

Since the auxiliary method is entirely new, and designed specifically to alleviate ghost-region extrapolation, we present a verification example in this section based on a transient convectiondiffusion problem with a moving interface. We solve the 1D transient convection-diffusion equation in each fluid zone i.e.:

$$
\frac{\partial \phi_{l}}{\partial t}=D_{l} \frac{\partial^{2} \phi_{l}}{\partial x^{2}}-u \frac{\partial \phi_{l}}{\partial x} \quad l=1,2
$$

Where $D_{l}$ is the thermal diffusivity, $u$ is the fluid velocity and $l$ is the zone. We enforce continuity of flux and solution at the moving interface which is convected with the fluid velocity $u$ :

$$
\begin{aligned}
\phi_{1} & =\phi_{2}, \\
D_{1} \frac{\partial \phi_{1}}{\partial x} & =D_{2} \frac{\partial \phi_{2}}{\partial x}
\end{aligned}
$$

At the boundaries $(-L \leq x \leq L)$, Dirichlet boundary conditions are enforced:

$$
\begin{aligned}
\phi_{1}(x=-L, t) & =1 \\
\phi_{2}(x=L, t) & =0
\end{aligned}
$$

The initial conditions for the problem, with the interface located at $-L / 2$ are:

$$
\begin{aligned}
& \phi_{1}(x \leq-L / 2,0)=1 \\
& \phi_{2}(x>-L / 2,0)=0
\end{aligned}
$$

The auxiliary multi-zone method can be used to solve the above problem, with the nodal points near the interface displaced at each time step to lie directly on the interface. PDE operators close to the interface are similarly displaced so that at each time step they have available data from the same zone at the previous time step. Since there are also no PDE operators directly on the interface, no extrapolation has to be performed for any PDE operators, with the nodal points being suitable displaced due to the meshless nature of the scheme. We tested the above problem using the embedded multi-zone method with $1^{\text {st }}$ and $2^{\text {nd }}$ order Taylor series extrapolation (since PDE points have to exist on the interface for global accuracy), however, the extrapolation severely limited the accuracy of the scheme and made it ineffective for moving interface problems in its current form. We therefore only present results from the auxiliary problem here.

Although analytical solutions exist for various transient convection-diffusion type problems, the solutions are typically hard to constuct and involve complex functions of exponentials, Fourier series and infinite sums (see for example $[39,40]$ ). These solutions become even harder to construct for moving interface, multi-zone transient convection-diffusion problems, even with constant coefficients. Therefore, to verify the performance of the auxiliary problem, we transform the above moving multi-zone convection-diffusion problem using a moving reference system with velocity $u$ 
[41]. We introduce the transformed variables as:

$$
X^{*}=x-u t, \quad t^{*}=t
$$

Using these variables, the convection-diffusion problem (41) reduces to the following diffusion problem:

$$
\frac{\partial \phi_{l}}{\partial t^{*}}=D_{l} \frac{\partial^{2} \phi_{l}}{\partial X^{* 2}} \quad l=1,2
$$

We solve equation (49) on a static frame from $\left(-L \leq X^{*} \leq L\right)$, and map the results back to the original $x$ domain. Since the newly mapped points in $x$ will be displaced to the right of the original domain, i.e. $-L+u t \leq x \leq L+u t$, any points that lie $-L \leq x \leq-L+u t$ are set to the boundary condition at $x=-L$. This is valid as long as the Peclet number is much greater than 1 meaning the solution near the left boundary at $x=-L$ will remain at the Dirichlet boundary condition and simply be convected into the domain. Any points that map to the region outside of the original domain, i.e., $x \geq L$ are not used. The mapped and original results will only be comparable if the solution at the points $X^{*} \geq L-u t$ is equal to the boundary condition at $x=L$. As long as the solution profile is sharp, the solution at the right of the domain remains near zero for a long time, allowing the solutions to be compared. For this procedure to work, the boundary conditions for the fixed $X^{*}$ domain have to be interpolated from the mapped domain, i.e.:

$$
\begin{aligned}
\phi_{1}\left(X^{*}=-L, t^{*}\right) & =\phi_{1}(x=-L+u t, t) \\
\phi_{2}\left(X^{*}=L, t^{*}\right) & =\phi_{2}(x=L+u t, t)=0
\end{aligned}
$$

Since the interface is displacing in time with position $x=-L / 2+u t$, the transformed matching conditions are:

$$
\begin{aligned}
\phi_{1} & =\phi_{2}, \\
D_{1} \frac{\partial \phi_{1}}{\partial X^{*}} & =D_{2} \frac{\partial \phi_{2}}{\partial X^{*}} \quad \text { at } \quad X^{*}=-L / 2
\end{aligned}
$$

Finally the initial conditions for the transformed problem are:

$$
\begin{aligned}
& \phi_{1}\left(X^{*} \leq-L / 2,0\right)=1 \\
& \phi_{2}\left(X^{*}>-L / 2,0\right)=0
\end{aligned}
$$

Solving equation (49) subject to boundary, matching and initial conditions (50) to (55) and mapping the results to the domain $x=X^{*}+u t$ following the assumptions above, is equivalent to solving (41) subject to boundary, matching and initial conditions (44) to (47) on the original $x$ domain.

Although the solution to equation (49) can be sought analytically (e.g. [42, 43]), the analytical solutions typically involve eigenvalue problems, whose solution is usually sought numerically. In the case here, we use a very fine resolution finite difference scheme to solve the governing equation (49) directly. Equation (49) is solved using finite differences (FD) on the domain $\left(-2 \leq X^{*} \leq 2\right)$. We use $2^{\text {nd }}$ order central differencing for the spatial derivatives, and a $1^{\text {st }}$ order fully implicit time stepping procedure for the temporal derivatives. A ghost node either side of the interface is used to close the system of equations arising from the extra flux and solution matching conditions at the interface. Initially the interface is located at $x=-1$, which with a velocity of $u=1$ and simulation time of $t=1$ results in an end location at $x=0$. To achieve a spatial and temporally independent solution, we use 511 solution nodes in the $\mathrm{x}$ direction with a time step size of $\Delta t=1 \times 10^{-7}$. Even though implicit time stepping is used, the boundary conditions must be taken at the previous time step where the mapped solution is known (it is not known at the current time when the solution is sought).

Equation (41) is solved using the auxiliary multi-zone RBF-FC method on the domain $(-2 \leq$ $x \leq 2)$. We use several different nodal discretisations, with a time step size of $\Delta t=1 \times 10^{-3}$. The same velocity field and starting interface location are used as above. We use a non-dimensional 


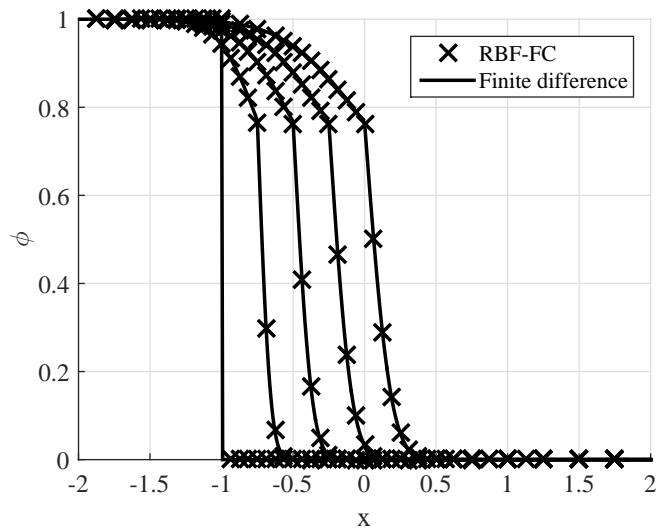

(a) $D_{1}=0.10, D_{2}=0.01$.

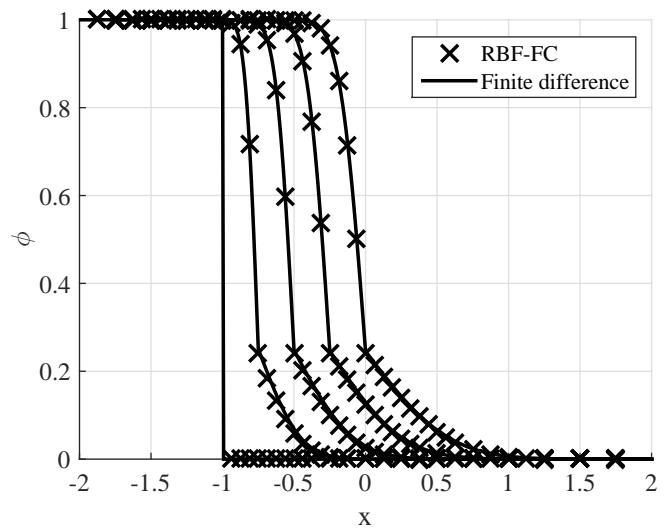

(b) $D_{1}=0.01, D_{2}=0.10$.

Figure 15: Moving interface solution profiles. RBF-FC solutions taken using quadtree cell level 4-6 dataset at the centreline $\mathrm{y}=0$, average 40.37 nodes in $\mathrm{x}$ direction. Solutions shown at $\Delta t=0.25$ increments from $0 \leq t \leq 1$.

shape parameter of $c^{*}=50$. The one-dimensional problem is solved in two dimensional space with the RBF method, using zero flux conditions at the top and bottom boundaries to ensure a one dimensional solution.

In the results that follow, the finite difference and RBF solutions are compared at time $t$ and spatial locations $x$, meaning the finite difference solution has to be mapped to the original domain, i.e. $x=X^{*}+u t$, with the approximations above.

Figure 15 shows the auxiliary RBF-FC and FD method solution profiles for two diffusivity regimes with time. Both solutions show sharp multi-zone interfaces where the diffusivity ratio causes a corresponding transition in the solution gradient. Since the interface moves with the convective field, the transient profile does not show the usual solution trapping at the discontinuity common in fixed interface problems [33]. Here, we see that as the inner zone expands, the solution cannot stagnate near the interface and increase with time, instead the solution is 'swept' along with the interface. In this way, once the transient profile has been setup after $t=0.25$, the solution at the interface does not change significantly, as any increase through convection/diffusion is matched by an expanding zone acting to lower the temperature.

The RBF solution in Figure 15 shows a highly accurate solution profile that is indistinguishable from the FD profile, with over 12 times fewer nodal points. The FD profile represents a highly accurate solution to the problem, since it solves a simple diffusion case with changing boundary conditions that is then simply mapped to the original domain. The RBF method has to update the interface location and displace the nodal points at every step, but is able to accurately capture the solution with very few points.

\begin{tabular}{|c|c|c|c|c|}
\hline Quadtree cell Level & Average Nx & Average $\Delta$ & $\begin{array}{c}L_{2} \text { difference } \\
\text { Case 1 }\end{array}$ & $\begin{array}{c}L_{2} \text { difference } \\
\text { Case 2 }\end{array}$ \\
\hline Uniform level 5 & 31 & 0.1250 & $4.01 \times 10^{-3}$ & $5.69 \times 10^{-3}$ \\
\hline Uniform level 6 & 63 & 0.0625 & $1.27 \times 10^{-3}$ & $2.14 \times 10^{-3}$ \\
\hline Quadtree level 4-6 & 40.37 & 0.0967 & $1.59 \times 10^{-3}$ & $2.60 \times 10^{-3}$ \\
\hline
\end{tabular}

Table 5: Moving interface relative errors between the auxiliary multi-zone RBF-FC method and the FD method at $t=1$.

The solution differences taken at $t=1$ between the FD and RBF-FC method for different datasets are shown in Table 5. As expected, the uniform level 6 RBF dataset shows the lowest difference, with a significantly refined mesh. Due to the relatively coarse time step size used, the errors do not drop significantly with an increase in the maximum cell level, since the error is controlled by the $2^{\text {nd }}$ order Crank-Nicholson time marching scheme. The error in the level 4-6 $\mathrm{RBF}$ dataset is very close to the uniform level 6 dataset, despite the significantly reduced number of nodes. Since the solution field changes sharply at the interface, very few nodes can be used in 
the outer domain, whilst nodes are clustered around the interface.

These results highlight the highly robust nature of the auxiliary multi-zone method for moving multi-zone problems. The solutions errors are low when compared to a transformed FD solution using only very few nodal points. Without extrapolation the scheme is also able to accurately capture the interface solution and surrounding solution profile, that make it very robust and not limited in spatial convergence to the extrapolation order. This represents a large improvement over fixed mesh schemes that have to use extrapolation when the interface moves over the nodes, and makes it very robust for the moving interface problems that are tackled in this work. 